\title{
Colour reconnections in Herwig++
}

\author{
Stefan Gieseke ${ }^{1, \mathrm{a}}$, Christian Röhr ${ }^{1, \mathrm{~b}}$, Andrzej Siódmok ${ }^{1,2, \mathrm{c}}$ \\ ${ }^{1}$ Institut für Theoretische Physik, Karlsruhe Institute of Technology (KIT), Karlsruhe, Germany \\ ${ }^{2}$ Consortium for Fundamental Physics, School of Physics and Astronomy, The University of Manchester, Manchester, UK
}

Received: 16 October 2012 / Revised: 24 October 2012 / Published online: 13 November 2012

(C) The Author(s) 2012. This article is published with open access at Springerlink.com

\begin{abstract}
We describe the implementation details of the colour reconnection model in the event generator HERWIG++. We study the impact on final-state observables in detail and confirm the model idea from colour preconfinement on the basis of studies within the cluster hadronization model. Moreover, we show that the description of minimum bias and underlying event data at the LHC is improved with this model and present results of a tune to available data.
\end{abstract}

\section{Introduction}

High-energy hadronic collisions at the Large Hadron Collider (LHC) require a sound understanding of soft aspects of the collisions. All hard collisions are accompanied by the underlying event (UE) which adds hadronic activity in all phase space regions. The physics of the underlying event is similar to the physics in minimum bias (MB) interactions and very important to understand to quantify the impact of pile-up in high-luminosity runs at the LHC. A wide range of measurements at the Tevatron and the LHC gives us a good picture of MB interactions and the UE [1-13]. Data has also shown that a good part of the underlying event is due to hard multiple partonic interactions (MPI). By now, the three major Monte Carlo event generators Herwig [14], PYTHIA $[15,16]$ and SHERPA [17] have an MPI model implemented to simulate the underlying event.

Such a model of independent multiple partonic interactions was first implemented in PYTHIA [18] where its relevance for a description of hadron collider data was immediately shown. On a similar physics basis, but with some differences in the detailed modelling the JIMMY add-on to the old HERWIG program, was introduced [19]. In these models, the average number of additional hard scatters is calculated

\footnotetext{
a e-mail: stefan.gieseke@kit.edu

b e-mail: christian.roehr@kit.edu

c e-mail: andrzej.siodmok@manchester.ac.uk
}

from a few input parameters and then for each hard event the additional number of hard scatters is sampled. The individual scatters in turn are modelled similarly to the primary hard scatters from QCD $2 \rightarrow 2$ interactions at leading order, with parton shower and hadronization applied as usual. The current underlying event model in SHERPA [17] is similar but will be replaced by a new approach [20]. The current model in PYTHIA differs from the original development in some details and follows the idea of interleaved partonic interactions and showering [21,22].

In the recent releases of HERWIG an MPI model is also included [23]. It comes with two main parameters, the minimum transverse momentum $p_{\perp}^{\mathrm{min}}$ of the additional hard scatters and the parameter $\mu^{2}$, that can be understood as the typical inverse proton radius squared and appears in the spatial transverse overlap of the incoming hadrons. Good agreement with Tevatron data was found with this model. Soft interactions were added to this model in order to improve consistency with more general theoretical input as the total cross section and the elastic slope parameter in high-energy hadronic collisions [24]. The distribution of transverse momenta in the non-perturbative region below $p_{\perp}^{\min }$ was modelled similarly to the proposal in [25]. Furthermore, it is assumed that the soft partons are distributed differently from the hard partons inside the hadron. The additional parameters introduced here are fixed by requiring a description of the total cross section and the slope parameter, so we are still left with only two parameters. Once again, a good description of Tevatron data on the UE was found, now also where softer interactions play a role. The model for soft interactions smoothly extrapolates from the perturbative into the non-perturbative region, similar to a model for intrinsic transverse momentum in initial-state radiation [26].

With the advent of new data from the LHC at $900 \mathrm{GeV}$ [3] we also considered new observables and found distinct disagreement with data, e.g. in the pseudorapidity of charged particles. It was clear that our implementation was incomplete as we have not at all tried to modify the relative colour 
structure of the multiple hard scatters. In Fig. 1 we show the sensitivity to the parameter $p_{\text {disrupt }}$, which controls the colour structure of soft scatters and see a partial refill of the central rapidity plateau. This notable dependence on $p_{\text {disrupt }}$ of soft scatters hints at the importance of colour correlations in a more complete model. Furthermore, we studied the dependence on other possible sources, e.g. on the parton distribution functions (PDF), which are used to extract the additional partons from the hadrons. In Fig. 2 we show the pseudorapidity of charged particles and the average transverse momentum as a function of particle multiplicity, $\left\langle p_{\perp}\right\rangle\left(N_{\mathrm{ch}}\right)$, at that stage. The lines represent different settings of the parameter of soft colour disruption and two different PDF sets: CTEQ6L1 [27] and MRST LO** [28]. We stress that all settings gave a good description of the Tevatron UE data. As discussed in more detail in [29-31], even a dedicated tuning of the MPI model parameters did not improve this description, which lead us to include a colour reconnection (CR) model in order to improve the colour structure between various hard scatters in the MPI model. The starting point is the idea of colour preconfinement [32]. While in a single hard interaction the colour structure is given by (the leading part of) the colour matrices that appear in the Feynman diagrams and also by the parton shower evolution, there is no such firm prescription for the assignment of colour lines or colour connections between individual hard scatters. Colour preconfinement leads us to the assumption that hard jets emerging from separate hard scatters should end up colourconnected when they are produced nearby in momentum space. As there is no such correlation in the non-perturbative modelling of the multiple hard interactions, we have to impose a model on it. Studies of such a model were carried out
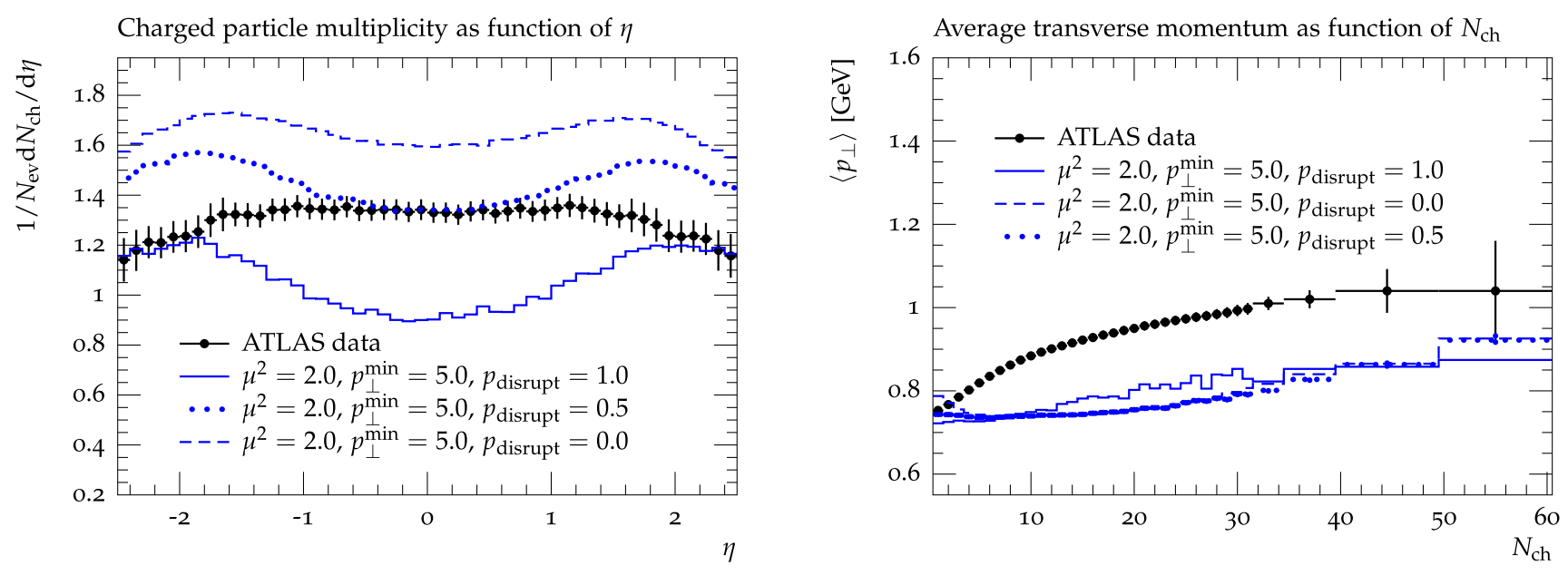

Fig. 1 Comparison of Herwig 2.4.2 (without CR) to ATLAS minimum-bias distributions at $\sqrt{s}=0.9 \mathrm{TeV}$ with $N_{\mathrm{ch}} \geq 2, p_{\perp}>500 \mathrm{MeV}$ and $|\eta|<2.5$. The HERWIG results are obtained by using three different values for $p_{\text {disrupt }}: 0.0,0.5$ and 1.0

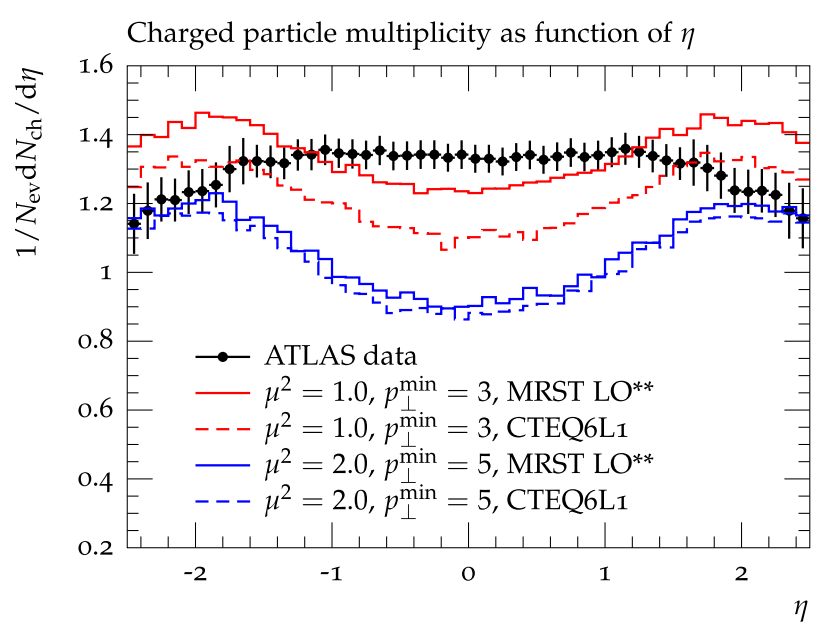

Fig. 2 Dependence on the choice of the PDF set. The shown observables are the same as already introduced in Fig. 1. We show results from two parameter points of the MPI model. For each point, two dif-

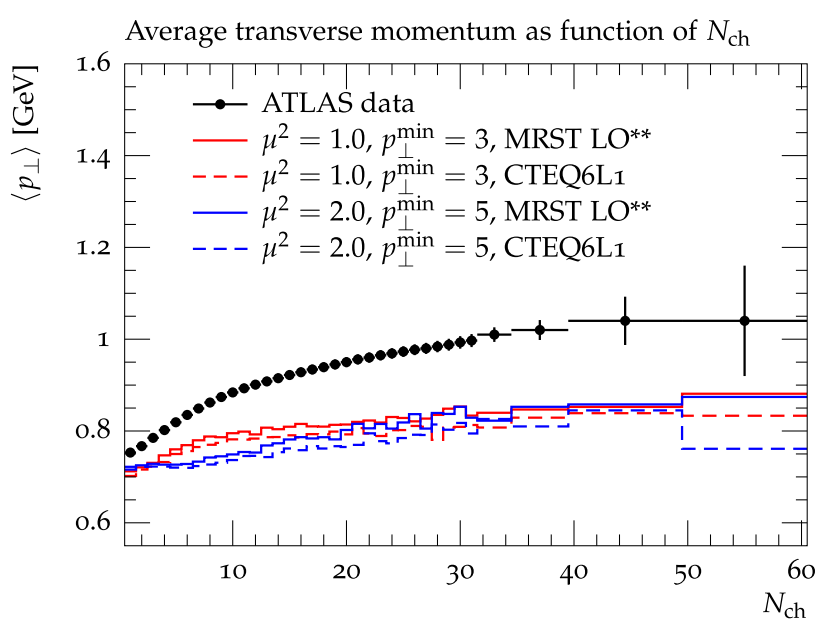

ferent PDF sets are selected, CTEQ6L1 and MRST LO**. All settings give a satisfactory description of the Tevatron underlying-event data 
earlier in [33-35]. In this paper we describe the details of such a colour reconnection model and confirm this physical picture with various analyses of the modelled hadronic final state. Finally, we present results of tuning this model to the currently available data on MB interactions and the UE.

\section{Modelling colour reconnections}

The cluster hadronization model [36] is based on planar diagram theory [37]: The dominant colour structure of QCD diagrams in the perturbation expansion in $1 / N_{\mathrm{c}}$ can be represented in a planar form using colour lines, which is commonly known as the $N_{\mathrm{c}} \rightarrow \infty$ limit. The resulting colour topology in Monte Carlo events with partons in the final state features open colour lines after the parton showers. Following a non-perturbative isotropic decay of any left gluons in the parton jets to light quark-antiquark pairs, the event finally consists of colour-connected partons in colour triplet or anti-triplet states. These parton pairs form colour-singlet clusters.

In dijet production via $e^{+} e^{-}$annihilation the invariant mass spectrum of these clusters is independent of the scale of the hard process $[36,38]$. The mass distribution peaks at small values, $\mathcal{O}(1 \mathrm{GeV})$, and quickly falls off at higher masses. Descriptively speaking, the cluster constituents tend to be close in momentum space. This property of perturbative QCD is referred to as colour preconfinement, as already stated above. The invariant cluster mass largely consists of the constituent rest masses, which gives rise to a pronounced peak at the parton rest mass threshold. Hence, clusters are interpreted as highly excited pre-hadronic states. In the cluster hadronization model hadrons normally arise from nonperturbative, isotropic cluster decays. The HERWIG implementation of this hadronization model is described in more detail in Ref. [14].

The situation in hadron collisions is necessarily more complicated. In a typical QCD $2 \rightarrow 2$ scatter, there is QCD radiation from the initial-state parton shower accompanied by jets emerging from outgoing partons. Due to colour charge conservation, there are colour connections between the partonic subprocess and the two hadron remnants. As sketched in Fig. 3, the primary hard subprocess is modelled in HERWIG as an interaction of two valence (anti)quarks [14]. Hence, in $p p(p \bar{p})$ collisions the hadron remnants are colour anti-triplets (triplets). The typical length scale of the valence parton extraction is the hadron size, $\mathcal{O}(1 \mathrm{fm})$, corresponding to energies where perturbation theory is not applicable. Thus, perturbative QCD cannot be used to calculate or assess the colour correlation between the partonic subprocess and the beam remnants.

We face a similar situation if we consider multiple parton interactions in single hadron collisions. The MPI model in HERWIG equips the event with a number of further QCD

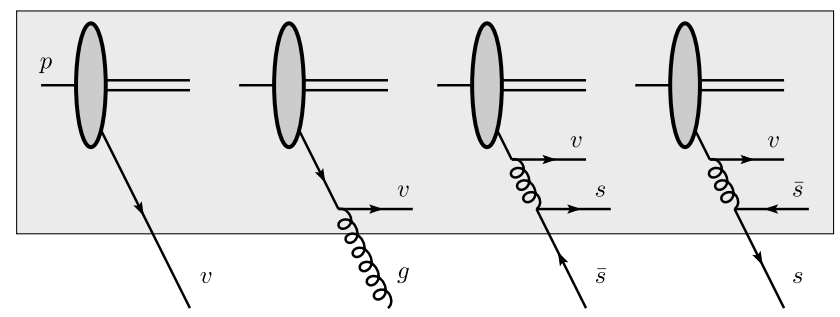

Fig. 3 For the hard subprocess a valence quark $v$ is extracted from the proton. Since the valence quark parton distribution functions dominate at large momentum fractions $x$ and small scales $Q^{2}$, the initial-state shower, which is generated backwards starting from the partonic scatter, commonly terminates on a valence quark. This situation is shown in the leftmost figure. If the perturbative evolution still terminates on a sea (anti)quark or a gluon, as indicated in the other figures, one or two additional non-perturbative splittings are performed to force the evolution to end with a valence quark. The grey-shaded area indicates this non-perturbative region, whereas the perturbative parton shower happens in the region below

parton scatters, in addition to the primary partonic subprocess. For each of these subprocesses a pair of gluons, initiating the scatter, is extracted from the colliding hadrons. The chosen colour topology for this extraction corresponds to the $N_{\mathrm{c}} \rightarrow \infty$ limit. As stated above, this limit is justified in perturbative branchings. In non-perturbative regimes, however, it is rather a QCD-motivated model than an assessable approximation.

As can be seen in the sketch in Fig. 7 below, the parton extraction model for the first and possible additional partonic subprocesses introduces colour lines, which connect subprocesses to each other and to the hadron remnants. As a result, clusters emerge in hadronic collisions which link different parts of the hadron collision. Clearly, these clusters cannot be expected to feature the same invariant-mass distribution as the clusters in $e^{+} e^{-}$dijet events do. Yet the cluster hadronization model for hadronic collisions is adopted unchanged. Colour reconnection intervenes at the stage right before hadrons are generated from the clusters. It provides the possibility to create clusters in a way which does not strictly follow the actual colour topology: The ends of the colour lines are reconnected, resulting in a different cluster configuration. This rearrangement of colour charges is pictorially shown in Fig. 4. Based on the successful role of preconfinement in $e^{+} e^{-}$collisions, we designed two colour reconnection models to work out colour singlets with invariant masses smaller than a priori given. The colour reconnection models studied in this paper differ in the underlying algorithm to find alternative cluster configurations.

\subsection{Plain colour reconnection}

A first model for colour reconnection has been implemented in HERWIG as of version 2.5 [39]. We refer to it as the plain colour reconnection model (PCR) in this paper. The following steps describe the full procedure: 


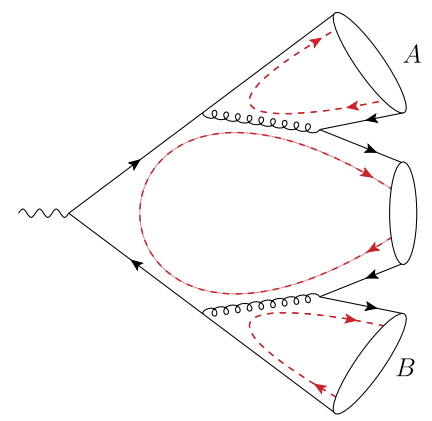

Fig. 4 Formation of clusters, which we represent by ovals here. Colour lines are dashed. The left diagram shows colour-singlet clusters formed according to the dominating colour structure in the $1 / N_{\mathrm{c}}$ ex-

1. Create a list of all quarks in the event, in random order. Perform the subsequent steps exactly once for every quark in this list.

2. The current quark is part of a cluster. Label this cluster $A$.

3. Consider a colour reconnection with all other clusters that exist at that time. Label the potential reconnection partner $B$. For the possible new clusters $C$ and $D$, which would emerge when $A$ and $B$ are reconnected (cf. Fig. 4), the following conditions must be satisfied:

- The new clusters are lighter,

$$
m_{C}+m_{D}<m_{A}+m_{B},
$$

where $m_{i}$ denotes the invariant mass of cluster $i$.

- $C$ and $D$ are no colour octets.

4. If at least one reconnection possibility could be found in step 3, select the one which results in the smallest sum of cluster masses, $m_{C}+m_{D}$. Accept this colour reconnection with an adjustable probability $p_{\text {reco. In this case }}$ replace the clusters $A$ and $B$ by the newly formed clusters $C$ and $D$.

5. Continue with the next quark in step 2 .

The parameter $p_{\text {reco }}$ steers the amount of colour reconnection in the PCR model. Because of the selection rule in step 4, the PCR model tends to replace the heaviest clusters by lighter ones. A priori the model is not guaranteed to be generally valid because of the following reasons: The random ordering in the first step makes this algorithm nondeterministic since a different order of the initial clusters, generally speaking, leads to different reconnection possibilities being tested. Moreover, apparently quarks and antiquarks are treated differently in the algorithm described above.

\subsection{Statistical colour reconnection}

The other colour reconnection implementation studied in this paper overcomes the conceptual drawbacks of the PCR

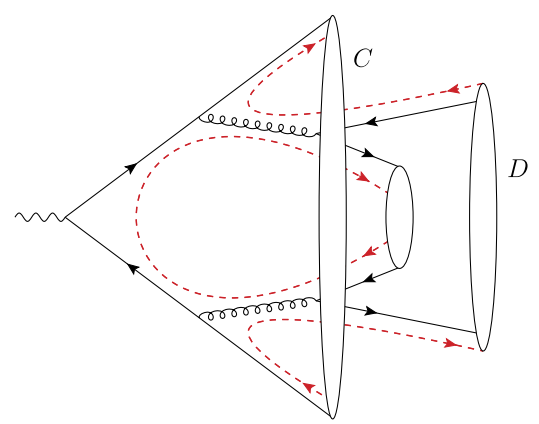

pansion. The right diagram shows a possible colour-reconnected state: the partons of the clusters $A$ and $B$ are arranged in new clusters, $C$ and $D$

model. We refer to this model as statistical colour reconnection (SCR) throughout this work. In the first place, the algorithm aims at finding a cluster configuration with a preferably small colour length, defined as

$\lambda \equiv \sum_{i=1}^{N_{\mathrm{cl}}} m_{i}^{2}$

where $N_{\mathrm{cl}}$ is the number of clusters in the event and $m_{i}$ is the invariant mass of cluster $i$. In the definition of the colour length we opt for squared masses to give cluster configurations with similarly heavy clusters precedence over configurations with less equally distributed cluster masses.

Clearly, it is impossible to locate the global minimum of $\lambda$, in general, since an event with 100 parton pairs, for instance, implies about $100 ! \approx 10^{158}$ possible cluster configurations to be tested. The Simulated Annealing algorithm from Ref. [40], however, has proven useful in solving optimisation problems like this approximately. The SCR model is an application of this algorithm with $\lambda$ as the objective function to be minimised.

The SCR algorithm selects random pairs of clusters and suggests them for colour reconnection. Just like in the PCR model, clusters consisting of splitting products of a colouroctet state are vetoed. A reconnection step which reduces $\lambda$ is always accepted. If the reconnection raises the colour length, it is accepted with probability

$p=\exp \left(-\frac{\lambda_{2}-\lambda_{1}}{T}\right)$,

where $\lambda_{1}$ and $\lambda_{2}$ denote the colour lengths before and after the reconnection, respectively. This gives the system the possibility to escape local minima in the colour length. The "temperature" $T$ is a control parameter, which is gradually reduced during the procedure. At high temperatures, $T \geq \mathcal{O}\left(\lambda_{2}-\lambda_{1}\right)$, the algorithm is likely to accept steps which raise $\lambda$. By contrast, lower temperatures imply a small probability for colour-length-increasing reconnection steps. 
The transition from high to low temperatures is determined by the annealing schedule, which flexibly adapts to the number of clusters, $N_{\mathrm{cl}}$, and to the colour length in the event. First, a starting temperature is determined from the typical change in the colour length, $\Delta \lambda=\lambda_{2}-\lambda_{1}$. To this end, a few random dry-run colour reconnections $S$ are performed, all starting with the default cluster configuration. The initial temperature is set to

$T_{\text {init }} \equiv c \cdot \operatorname{median}_{i \in S}\left\{|\Delta \lambda|_{i}\right\}$,

where $c$ is a free parameter of the model. Using the median makes this definition less prone to outliers compared to the mean. The algorithm proceeds in steps with fixed temperature. At the end of each temperature step $T$ decreases by a factor $f$, which is another free model parameter, with $f \in(0,1)$. Each value of $T$ is held constant for $\alpha N_{\mathrm{cl}}$ reconnection attempts with another free parameter $\alpha$. The algorithm stops as soon as no successful colour reconnections happen in a temperature step, but at most $N_{\text {steps }}$ temperature steps are tested. We use the parameters $c, \alpha, f$ and $N_{\text {steps }}$, which are all related to the annealing schedule, to tune the SCR model to data.

We would like to stress that the annealing model is used only as a numerical tool to minimize the colour length introduced above and hence give no physical interpretation to the model parameters themselves. We argue later, that merely the idea of minimizing the colour length is indeed meaningful and physical.

\section{Characteristics of colour reconnection}

In this section we want to study hadronization-related quantities which allow us to understand colour reconnection from an event generator-internal point of view. Here, a set of typical values for $c, \alpha, f$ and $N_{\text {steps }}$ in the SCR model, as well as for $p_{\text {reco }}$ in the PCR model, was used, which was obtained from tunes to experimental data, as described below in Sect. 4.

\subsection{Colour length drop}

To quantify the effect of colour reconnection at generator level, we define the colour length drop

$\Delta_{\text {if }} \equiv 1-\frac{\lambda_{\text {final }}}{\lambda_{\text {init }}}$,

where $\lambda_{\text {init }}$ and $\lambda_{\text {final }}$ denote the colour length in an event before and after colour reconnection, respectively. $\Delta_{\text {if }}$ approximately vanishes in events with $\lambda_{\text {init }} \approx \lambda_{\text {final }}$, i.e. with no or only minor changes in the colour length $\lambda$ due to colour reconnection. The other extreme, $\Delta_{\text {if }} \approx 1$, indicates a notable drop in $\lambda$.

The distribution of $\Delta_{\text {if }}$ for soft inclusive LHC events at $7 \mathrm{TeV}$ is shown in Fig. 5(a). The plain and the statistical colour reconnection models result in similar distributions with pronounced peaks at 0 and 1. Note that Fig. 5 shows logarithmic plots, so the plateau in between the peaks is really low. There is also a small fraction of events with negative $\Delta_{\mathrm{if}}$, though. The colour reconnection procedure actually raises $\lambda$ in these events. In the SCR algorithm, this can happen since $\lambda$-raising steps are explicitly allowed with a certain probability, cf. Eq. (3). However, also the PCR algorithm might potentially raise $\lambda$ since the reconnection condition, Eq. (1), is formulated in terms of the first power of cluster masses, whereas $\lambda$ is defined as the sum of squared cluster masses. As these events are rare, we expect no impact on physical observables.

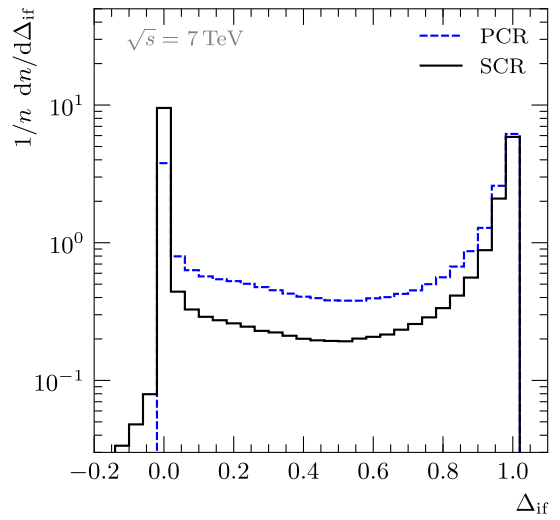

(a)

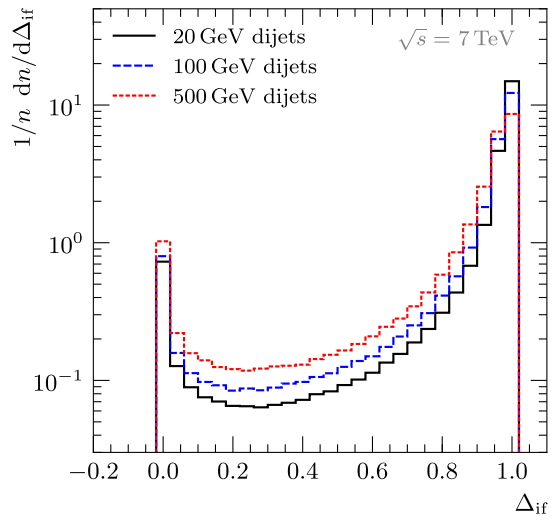

(b)

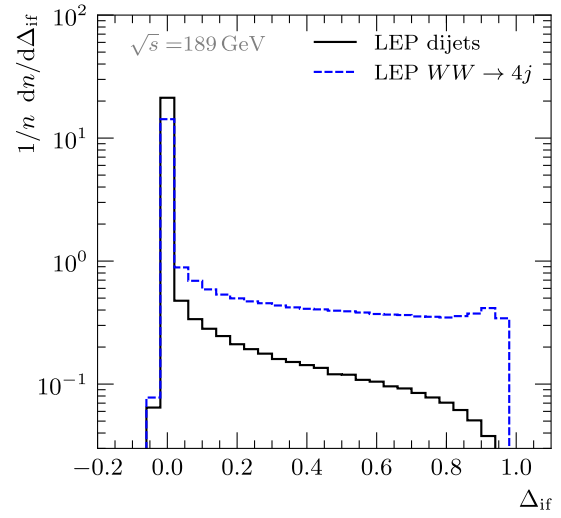

(c)
Fig. 5 Colour length drop in $p p$ and $e^{+} e^{-}$collisions. Figure (a) shows $\Delta_{\text {if }}$ using the PCR and the SCR models. The events were generated with soft inclusive LHC generator settings at $7 \mathrm{TeV}$. In (b) we show the colour length drop within the SCR model in LHC dijet produc- tion with a number of $p_{\perp}$ cuts, where the c.m. energy is also $7 \mathrm{TeV}$. (c) shows the drop in the colour length (using the SCR model) with LEP generator setup running at $189 \mathrm{GeV}$. We compare dijet events to $W$ boson pair production with fully hadronic decays 
With soft inclusive hadron-hadron generator settings there are, generally speaking, two important classes of events. One of the two are events where there is no notable change in the sum of squared cluster masses, $\lambda$. In another large fraction of events, however, colour reconnection causes an extreme drop in $\lambda$. An obvious interpretation for this drop is that the colour reconnection procedure replaces disproportionally heavy clusters by way lighter ones.

This shift in the cluster mass spectrum, which both models aim at by construction, can also be observed directly. Figure 6 shows the cluster mass distribution before and after colour reconnection. As expected and also intended, both $\mathrm{CR}$ procedures cause the distribution to be enhanced in the low-mass peak region and suppressed in its, potentially unphysical, high-mass tail.

In Fig. 5(b) we show the colour length drop in hard dijet events in $p p$ collisions. We observe a notable decrease of large colour length drops, $\Delta_{\text {if }}=1$, with increasing cut on the jet transverse momentum at parton level. The reason for this decrease is that higher momentum fractions are required for the hard dijet subprocess, whereas in soft events the remaining momentum fraction of the proton remnants is higher. Hence clusters containing a proton remnant are less massive in hard events, which implies less need for colour reconnection.

The distribution of the colour length drop in $e^{+} e^{-}$annihilation events looks completely different, as shown in Fig. 5(c). We find that colour reconnection has no impact on the colour length in the bulk of dijet events. We show only the $\Delta_{\text {if }}$ distribution from the SCR model here, but the PCR model yields similar results. These results confirm that due to colour preconfinement partons nearby in momentum space in most cases are combined to colour singlets already. In events with hadronic $W$ pair decays, however, hadrons emerge from two separate colour singlets. If there is a phase space overlap of the two parton jet pairs, the production of hadrons is expected to be sensitive to colour reconnection. We address this question later on in Sect. 4.1. Here we want to remark that the fraction of $W W$ events with nonvanishing colour length drop is slightly higher than for the dijet case. Nevertheless, the vast majority of $W W$ events is not affected by colour reconnection, too.

\subsection{Classification of clusters}

These results generically raise the question which mechanism in the hadron event generation is responsible for these overly heavy clusters. To gain access to this issue, we classify all clusters by their ancestors in the event history. A sketch of the three types of clusters in shown in Fig. 7.

- The first class are the clusters consisting of partons emitted perturbatively in the same partonic subprocess. We call them $h$-type (hard) clusters.

- The second class of clusters are the subprocesses-interconnecting clusters, which combine partons generated perturbatively in different partonic subprocesses. They are labelled as $i$-type (interconnecting) clusters.

- The remaining clusters, which can occur in hadron collision events, are composed of at least one parton created non-perturbatively, i.e. during the extraction of partons from the hadrons or in soft scatters. In what follows, these clusters are called n-type (non-perturbative) clusters.

First we use this classification to analyse hadron collision events as they are immediately before colour rearrangement. For that purpose, we define the cluster fraction functions

$f_{a}\left(m_{\mathrm{cut}}\right) \equiv N_{a}\left(m_{\mathrm{cut}}\right) / \sum_{b=h, i, n} N_{b}\left(m_{\mathrm{cut}}\right)=\frac{N_{a}\left(m_{\mathrm{cut}}\right)}{N_{\mathrm{cl}}}$,

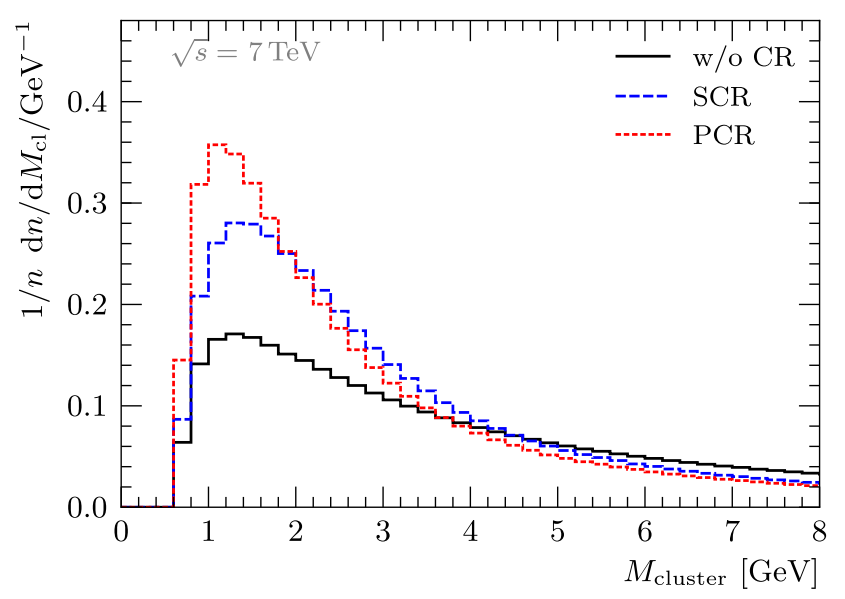

(a)

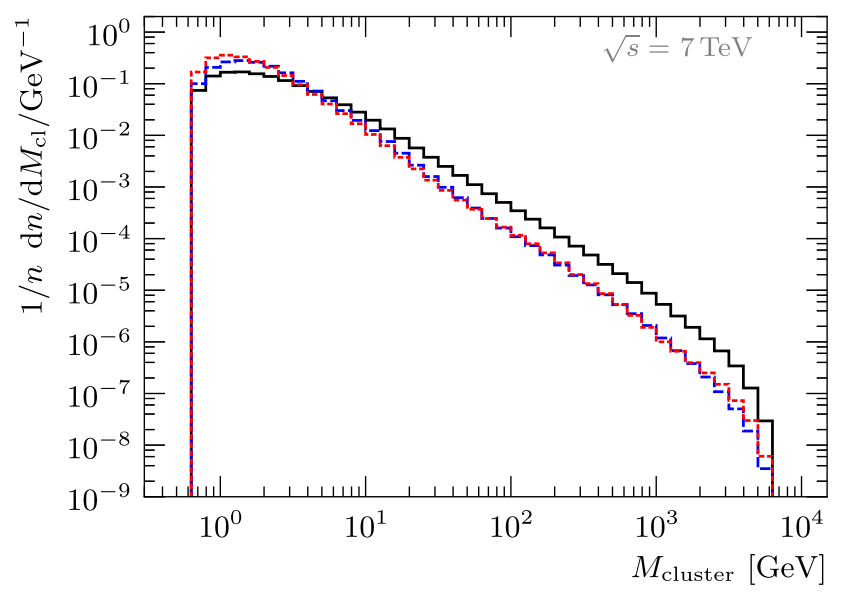

(b)

Fig. 6 Invariant mass of primary clusters in soft inclusive LHC events at $7 \mathrm{TeV}$. The histograms are normalized to unity, where also invisible bins are taken into account. The histograms in (b) differ from the ones in (a) only in their binning 
where $N_{a}\left(m_{\text {cut }}\right)$ is the number of $a$-type clusters ( $a=$ $h, i, n)$ with $m \geq m_{\text {cut }}$, counted in a sufficiently large number of events. ${ }^{1}$ For instance, $f_{i}(100 \mathrm{GeV})=0.15$ says $15 \%$ of all clusters with a mass larger than $100 \mathrm{GeV}$ are subprocess-interconnecting clusters. By construction, $f_{a}\left(m_{\text {cut }}\right)$ is a number between 0 and 1 for every class $a$. Moreover, the cluster fraction functions satisfy

$$
\sum_{a=h, i, n} f_{a}\left(m_{\mathrm{cut}}\right)=1
$$

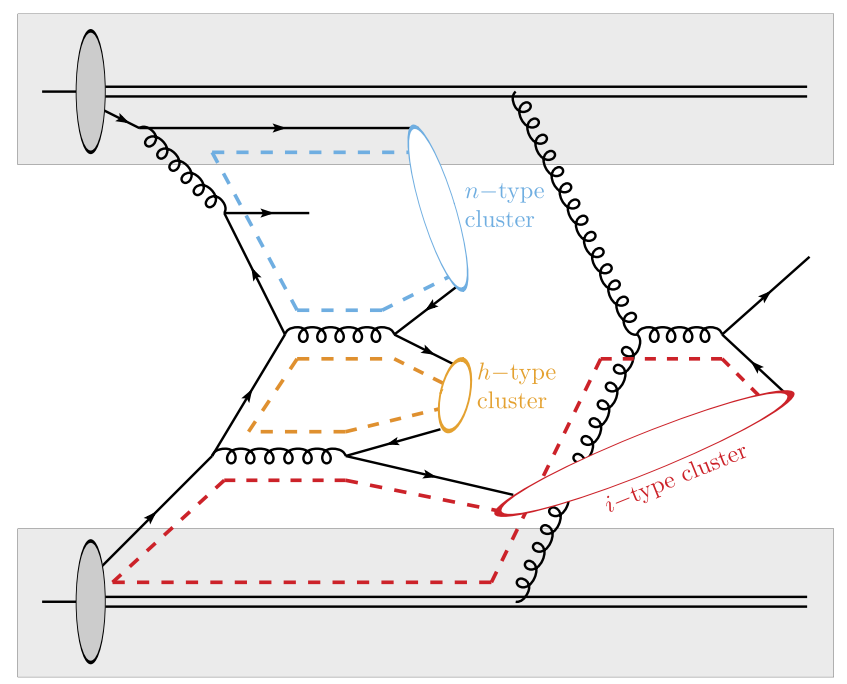

Fig. 7 Classification of colour clusters in a hadron collision event, which, in this example, consists of the primary subprocess (left) and one additional parton interaction. The grey-shaded area denotes non-perturbative parts of the simulation. The three clusters represent the cluster classes defined in Sect. 3.2: $n$-type, $i$-type and $h$-type clusters

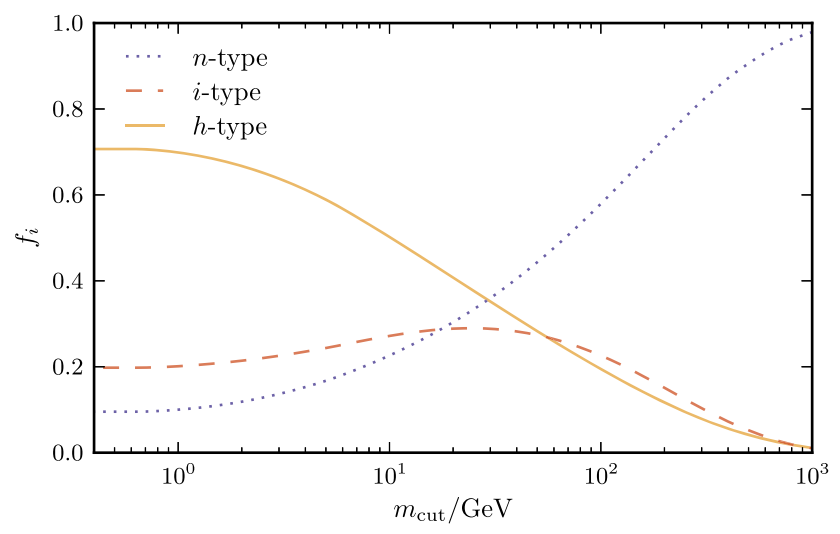

Fig. 8 Cluster fraction functions, defined in Eq. (6), for LHC dijet events at $7 \mathrm{TeV}$

\footnotetext{
${ }^{1}$ Apparently, $f_{a}\left(m_{\text {cut }}\right)$ is only well-defined for $m_{\text {cut }}$ less than the maximum cluster mass. On this interval, the series $\left(f_{a, n}\right)$, with $n$ the number of events taken into account, converges pointwise to the function $f_{a}$. This is a more formal definition of the cluster fraction functions.
}

Figure 8 shows the cluster fraction functions for LHC dijet events at $\sqrt{s}=7 \mathrm{TeV}$. The fraction of non-perturbative clusters increases with $m_{\text {cut }}$ and exceeds 0.5 at $m_{\text {cut }} \approx 70 \mathrm{GeV}$. So for an increasing threshold $m_{\text {cut }}$ up to values well beyond physically reasonable cluster masses of a few $\mathrm{GeV}$, the contribution of $n$-type clusters becomes more and more dominant.

A bin-by-bin breakdown to the contributions of the various cluster types to the total cluster mass distribution is shown in Fig. 9. There are several things to learn from those plots. First, non-perturbative $n$-type clusters do not contribute as much to the peak region, say below $6 \mathrm{GeV}$, as perturbative $h$-type and $i$-type clusters do. In the high-mass tail, however, $n$-type clusters clearly dominate, as already indicated by the cluster fraction functions discussed above. Both their minor contribution at low masses and their large contribution at high masses do not change after colour reconnection. In total, however, the mass distribution is more peaked after colour reconnection and the high-mass tail is suppressed by a factor larger than 10 .

\subsection{Resulting physics implications}

The characteristics of clusters that have been studied in this section clearly confirm the physical picture we have started out with. The colour reconnection model in fact reduces the invariant masses of clusters that are mostly of nonperturbative origin. These arise as an artefact of the way we colour-connect additional hard scatters in the MPI model with the rest of the event.

At this non-perturbative level we have no handle on the colour information from theory, hence we have modelled it. First in a very naïve way when we extract the 'first' parton from the proton, but only to account for a more physical picture later, where we use colour preconfinement as a guiding principle. We therefore conclude that our ansatz to model colour reconnections in the way we have done it reproduces a meaningful physical picture.

\section{Tuning and comparison of the model results with data}

In this section we address the question of whether the MPI model in HERWIG, equipped with the new CR model, can improve the description of the ATLAS MB and UE data, see Fig. 2. To that end we need to find values of free parameters (tune parameters) of the MPI model with CR that allow to get the best possible description of the experimental data. Since both CR models can be regarded as an extension of the cluster model [36], which is used for hadronization in HERWIG, the tune of HERWIG with CR models may require a simultaneous re-tuning of the hadronization model parameters to a wide range of experimental data, primarily from 
LEP (see Appendix D from Ref. [14]). Therefore, we start this section by examining whether the description of LEP data is sensitive to CR parameters.

\subsection{Validation against $e^{+} e^{-}$LEP data}

Already in Sect. 3 we have seen that the colour structure of LEP final states is well-defined by the perturbative parton shower evolution. Moreover, the CR model does not change this structure significantly. Therefore, although CR is an extension of hadronization, we can expect that the default hadronization parameters are still valid in combination with CR. This was confirmed by comparing HERWIG results with and without $\mathrm{CR}$ against a wide range of experimental data from LEP [41-49]. As an example we show a compari-

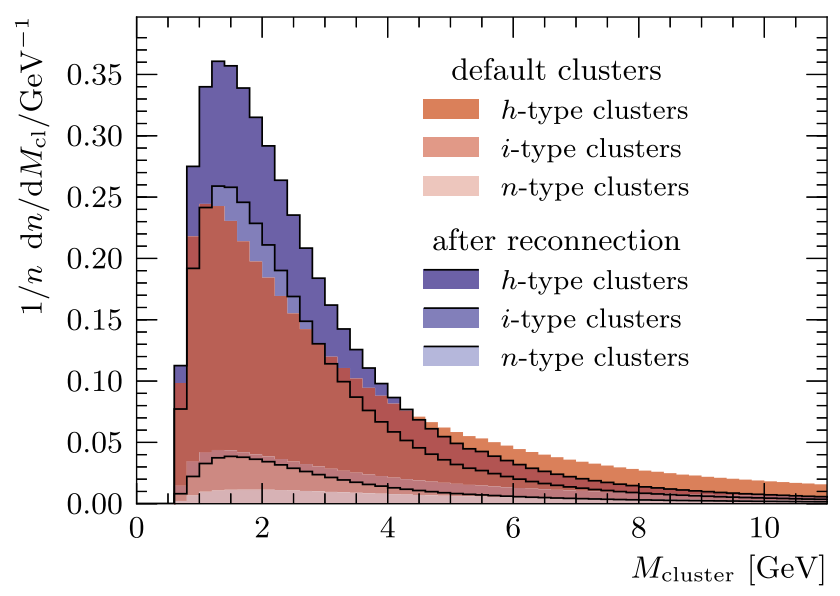

(a)

Fig. 9 Primary cluster mass spectrum in LHC dijet events at $7 \mathrm{TeV}$. Figure (a) compares the mass distribution in the pre-colourreconnection stage to the distribution after colour reconnection. The son of HERWIG without and with CR (using the main tunes for both CR methods presented in this paper) to two LEP observables in Fig. 10. The full set of plots, showing that the LEP data description in HERWIG with and without CR is of the same quality, can be found on the HERWIG and MCplots web pages $[50,51]$. These results allow us to factorize the tuning procedure: The well-tested default HERWIG tune for parton shower and hadronization parameters is retained, and only the parameters from the CR and MPI models are tuned to hadron collider data. However, we have checked each tune presented in this paper against LEP results.

In addition to the analyses used for the hadronization tuning, there are LEP analyses dedicated to colour reconnection in $W^{+} W^{-} \rightarrow(q \bar{q})(q \bar{q})$ events [52-55], originally proposed in Ref. [56]. In those analyses the $W$ bosons are

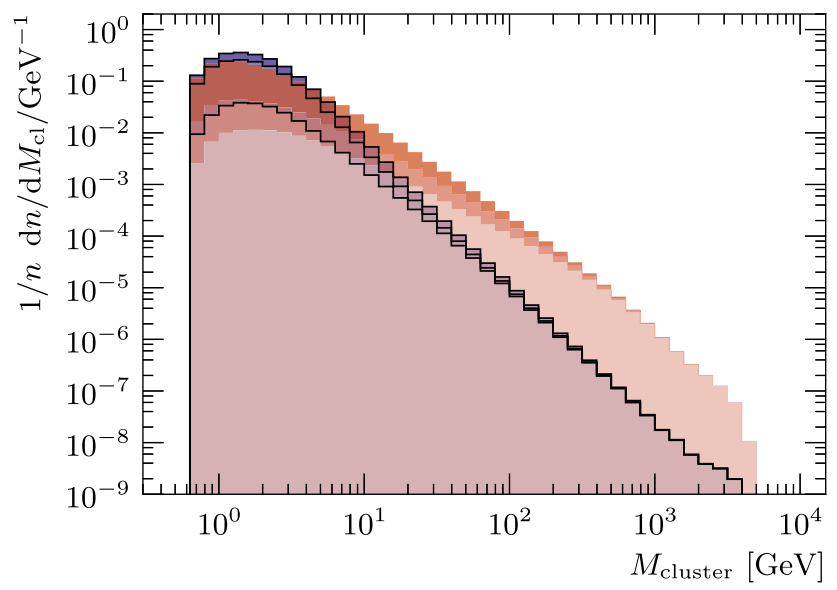

(b)

contributions of the three cluster classes are stacked. The histograms in (b) merely differ from the ones in (a) in their binning

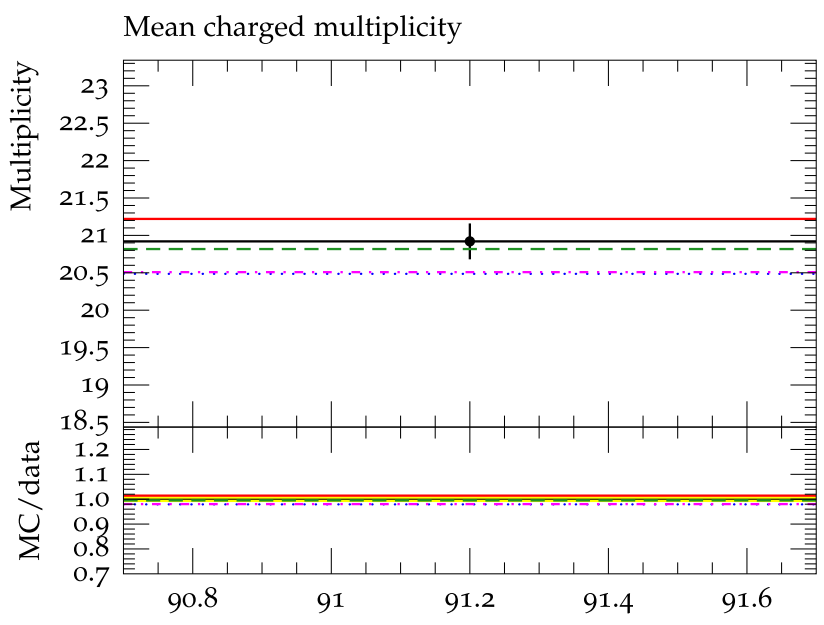

Fig. 10 Comparison of HERWIG without CR (solid red line) and with CR (using the main tunes for both CR methods presented in this paper) to exemplary measurements from the DELPHI detector at LEP 
reconstructed via kinematic cuts on all possible jet pairs in four-jet events. The particle flow between jets originating from different bosons was expected to be enhanced in Monte Carlo models including colour reconnection. However, only moderate sensitivity to the tested CR models could be found at the time. We have confirmed this with our colour reconnection implementations. In Fig. 11 we show the sensitivity of the particle flow between the identified jets to the reconnection strength in the PCR model, compared to DELPHI data from Ref. [52]. We observe a slight improvement in the description of the data. A number of apparent outliers in the experimental data, however, indicate possibly too optimistic systematic errors in the experimental analysis. For that reason, no clear constraints on the model can be deduced from the data.

As the $W$ bosons are produced on shell and significantly boosted at $\sqrt{s}=189 \mathrm{GeV}$, the finite $W$ width can cause the two $W$ bosons to travel long distances before decaying. In the limit of a very small $W$ width, large reconnection effects between the two $W$ systems should thus be suppressed in the model. The moderate sensitivity of the particle flow to colour reconnections implies, however, that colour reconnection effects are small in $W W$ events. Note that also the largely vanishing colour length drop in $W W$ events, cf. Fig. 5(c) and the discussion in Sect. 3.1, supports this conclusion. Hence we retain the described generic reconnection models also for $W W$ events and do not introduce an extra suppression mechanism.

\subsection{Tuning to data from hadron colliders}

Now that we have validated the CR models by comparison against LEP data, we are ready to tune their parameters to data provided by hadron colliders. Before LHC data was available, the MPI model in HERWIG [24] was tuned by

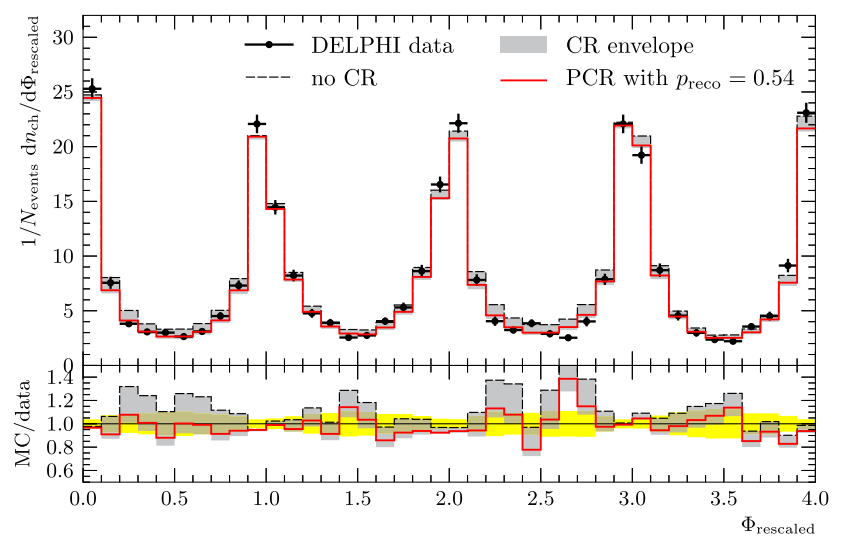

Fig. 11 Charged-particle flow in hadronic $W W$ events at LEP with $\sqrt{s}=189 \mathrm{GeV}$. The grey band indicates the range which is covered by varying the colour reconnection strength $p_{\text {reco }}$ in the PCR model. The definition of the rescaled angle, $\Phi_{\text {rescaled, }}$ along with a detailed description of the analysis can be found in Ref. [52] subdividing the two-dimensional parameter space, spanned by the model's main parameters, the inverse proton radius squared $\mu^{2}$ and the minimum transverse momentum $p_{\perp}^{\min }$, into a grid. For each of the parameter points on this grid, the total $\chi^{2}$ against the Tevatron underlying-event data $[1,57]$ was calculated. A region in the parameter plane was found, where similarly good values for the overall $\chi^{2}$ could be obtained.

While tuning the MPI models including colour reconnection we are dealing with a larger number $N$ of tunable parameters $p_{i}$, where $N=4$ in case of the PCR ( $p_{\text {disrupt }}, p_{\text {reco }}$, $p_{\perp}^{\min }$ and $\left.\mu^{2}\right)$ and $N=7$ in case of the SCR model ( $p_{\text {disrupt }}$, $p_{\perp}^{\min }, \mu^{2}, \alpha, c, f$ and $\left.N_{\text {steps }}\right)$. Hence the simple tuning strategy from above is ineffective. A comprehensive scan of 7 parameters, with 10 divisions in each parameter would require too much CPU time.

Instead, we use a parametrization-based tune method which is much more efficient for our case. The starting point for this tuning procedure is the selection of a range $\left[p_{i}^{\min }, p_{i}^{\max }\right]$ for each of the $N$ tuning parameters $p_{i}$. Event samples are generated for random points of this $\mathrm{N}$ dimensional hypercube in the parameter space. The number of different points depends on the number of input parameters to ensure a well converging behaviour of the final tune. Each generated event is directly handed over to the Rivet package [58] to analyse the generated events. This allows the computation of observables for each parameter point, which construct the input for the tuning process. The obtained distributions of observables for each parameter variation are the starting point for the main part of the tune, which is achieved using the Professor framework [59]. Professor parametrizes the generator response to the probed parameter points. In that way it finds the set of parameters, which fits the selected observables best. The user is able to affect the tuning by applying a weight for each observable, which specifies the impact of the variable for the tuning process.

\subsubsection{Tuning to minimum-bias data}

As we initially were primarily aiming at an improved description of MB data, we started by tuning the PCR model to ATLAS MB data. Since currently there is no model for soft diffractive physics in HERWIG, we use the diffractionreduced ATLAS MB measurement with an additional cut on the number of charged particles, $N_{\mathrm{ch}} \geq 6$. The observables we used for the tune are the pseudorapidity distribution of the charged particles, the charged multiplicity, the charged-particle transverse momentum spectrum and the average transverse momentum measured as a function of the number of charged particles. All four available MB observables entered the tune with equal weights. The results of this tune are shown by the blue lines in Fig. 12. The bottom right figure shows that colour reconnection helps to achieve 

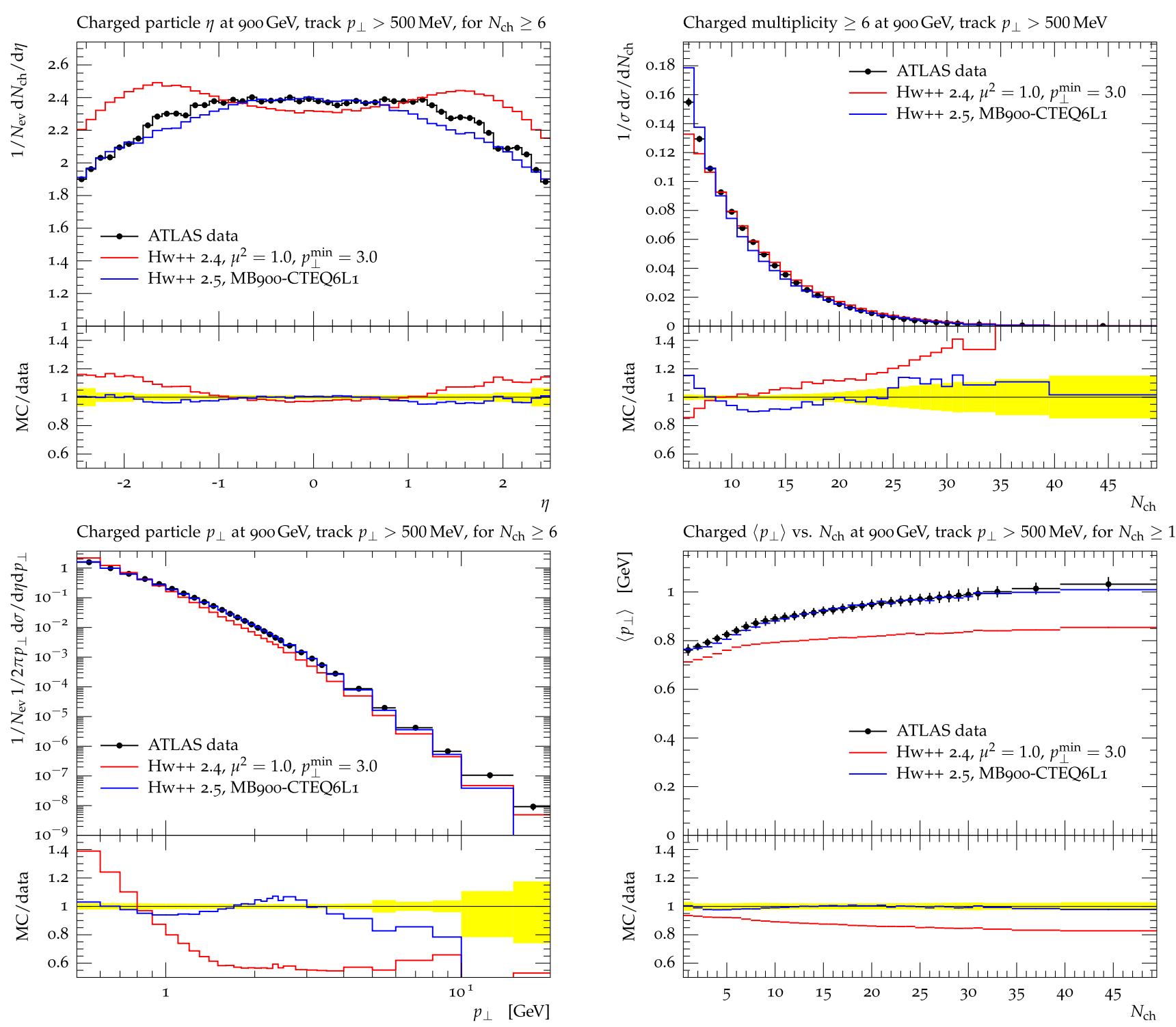

Fig. 12 Comparison of Herwig 2.4.2 without CR and HeRwig 2.5 with PCR to ATLAS minimum-bias distributions at $\sqrt{s}=0.9$ TeV with $N_{\mathrm{ch}} \geq 6, p_{\perp}>500 \mathrm{MeV}$ and $|\eta|<2.5$. The ATLAS data was published in Ref. [5]

a better description of $\left\langle p_{T}\right\rangle\left(N_{\mathrm{ch}}\right)$. Also the other three distributions are now well described. We conclude that the CR model was the missing piece of the MPI model in HERWIG++. We clearly improve the description of the pseudorapidity distribution.

\subsubsection{Tuning to underlying-event data}

The next important question was whether the new model is able to describe the UE data collected by ATLAS at $7 \mathrm{TeV}$ [4]. The measurements are made relative to a leading object (the hardest charged track in this case). Then, the transverse plane is subdivided in azimuthal angle $\phi$ relative to this leading object at $\phi=0$. The region around the leading object, $|\phi|<\pi / 3$, is called the "towards" region. The opposite re- gion, where we usually find a recoiling hard jet, $|\phi|>2 \pi / 3$, is called "away" region, while the remaining region, transverse to the leading object and its recoil, where the underlying event is expected to be least 'contaminated' by activity from the hard subprocess, is called "transverse" region. Again, we only focus on the tuning of the PCR model here. For the underlying-event tune two observables were used: The mean number of stable charged particles per unit of $\eta-\phi,\left\langle\mathrm{d}^{2} N_{\mathrm{ch}} / \mathrm{d} \eta \mathrm{d} \phi\right\rangle$, and the mean scalar $p_{\perp}$ sum of stable particles per unit of $\eta-\phi,\left\langle\mathrm{d}^{2} \sum p_{t} / \mathrm{d} \eta \mathrm{d} \phi\right\rangle$, both as a function of $p_{\perp}^{\text {lead }}$, with charged particles in the kinematic range $p_{\perp}>500 \mathrm{MeV}$ and $|\eta|<2.5$.

The resulting tune, named UE7-2, gives very satisfactory results not only for the tuned observables but also for all other observables provided by ATLAS in Ref. [4]. In 

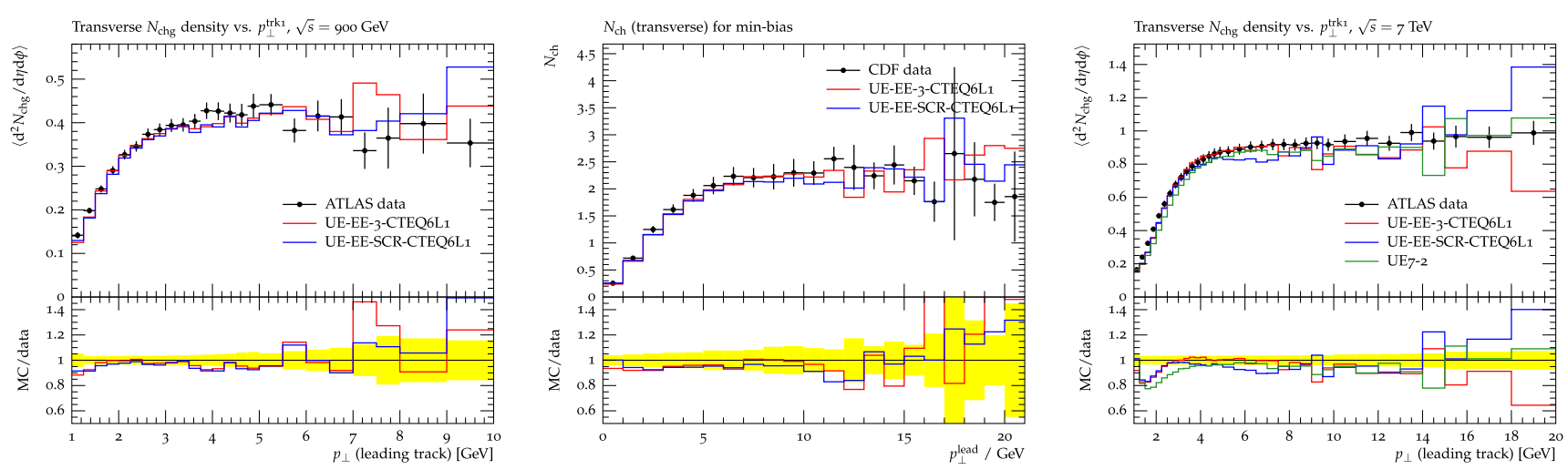

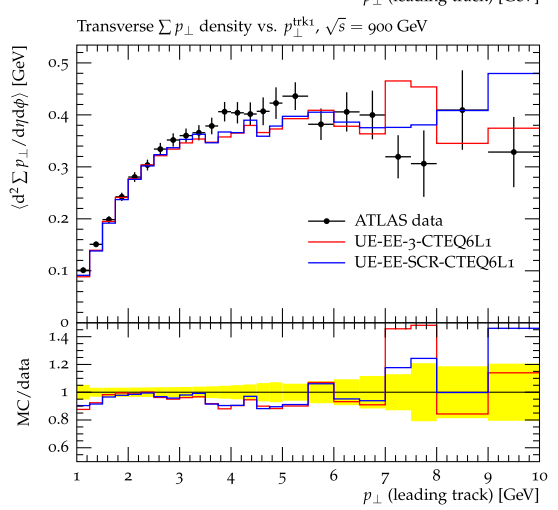

(a)

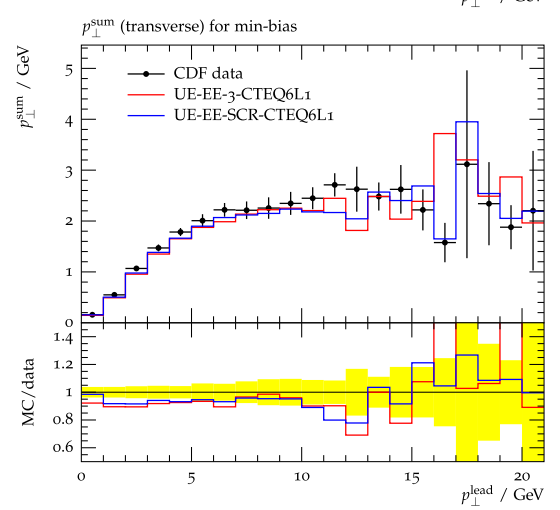

(b)

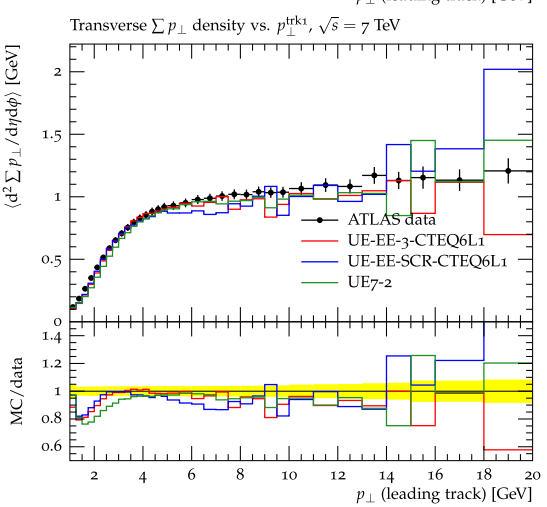

(c)
Fig. 13 ATLAS data at $900 \mathrm{GeV}$ (1 st column), CDF data at $1800 \mathrm{GeV}$ (2nd column) and ATLAS data at $7 \mathrm{TeV}$ (3rd column), showing the multiplicity density and $\sum p_{\perp}$ of the charged particles in the "trans- verse" area as a function of $p_{\perp}^{\text {lead }}$. The data is compared to the UE7-2, UE-EE-3-CTEQ6L1 and UE-EE-SCR-CTEQ6L1 tunes

At this stage different UE tunes were mandatory for different hadronic centre-of-mass energies $\sqrt{s}$. In the next section we address the question of whether an energy-independent UE tune can be obtained using the present model.

\subsubsection{Centre-of-mass energy dependence of UE tunes}

To study the energy dependence of the parameters properly, we examine a set of observables at different collider energies, whose description is sensitive to the MPI model parameters. The experimental data should be measured at all energies in similar phase-space regions and under not too different trigger conditions. These conditions were met by two UE observables: $\left\langle\mathrm{d}^{2} N_{\mathrm{ch}} / \mathrm{d} \eta \mathrm{d} \phi\right\rangle$ and $\left\langle\mathrm{d}^{2} \sum p_{t} / \mathrm{d} \eta \mathrm{d} \phi\right\rangle$, both measured as a function of $p_{\perp}^{\text {lead }}$ (with $p_{\perp}^{\text {lead }}<20 \mathrm{GeV}$ ) by ATLAS at 900 and $7000 \mathrm{GeV}$ (with $p_{\perp}>500 \mathrm{MeV}$ ) and by CDF at $1800 \mathrm{GeV}$. Let us first focus on the PCR model. In this case we have four free model parameters, $p_{\text {disrupt }}, p_{\text {reco }}$, $p_{\perp}^{\min }$ and $\mu^{2}$. For each hadronic centre-of-mass energy we performed independent four-dimensional tunings. Note that $p_{\perp}^{\text {lead }}$ denotes the transverse momentum of the hardest track in the case of ATLAS, whereas the CDF underlying-event analysis uses the $p_{\perp}$ of the leading jet, which we call $p_{\perp}^{\text {lead }}$ here, as well. and MB data sets is available on the HERWIG tune page [50]. 

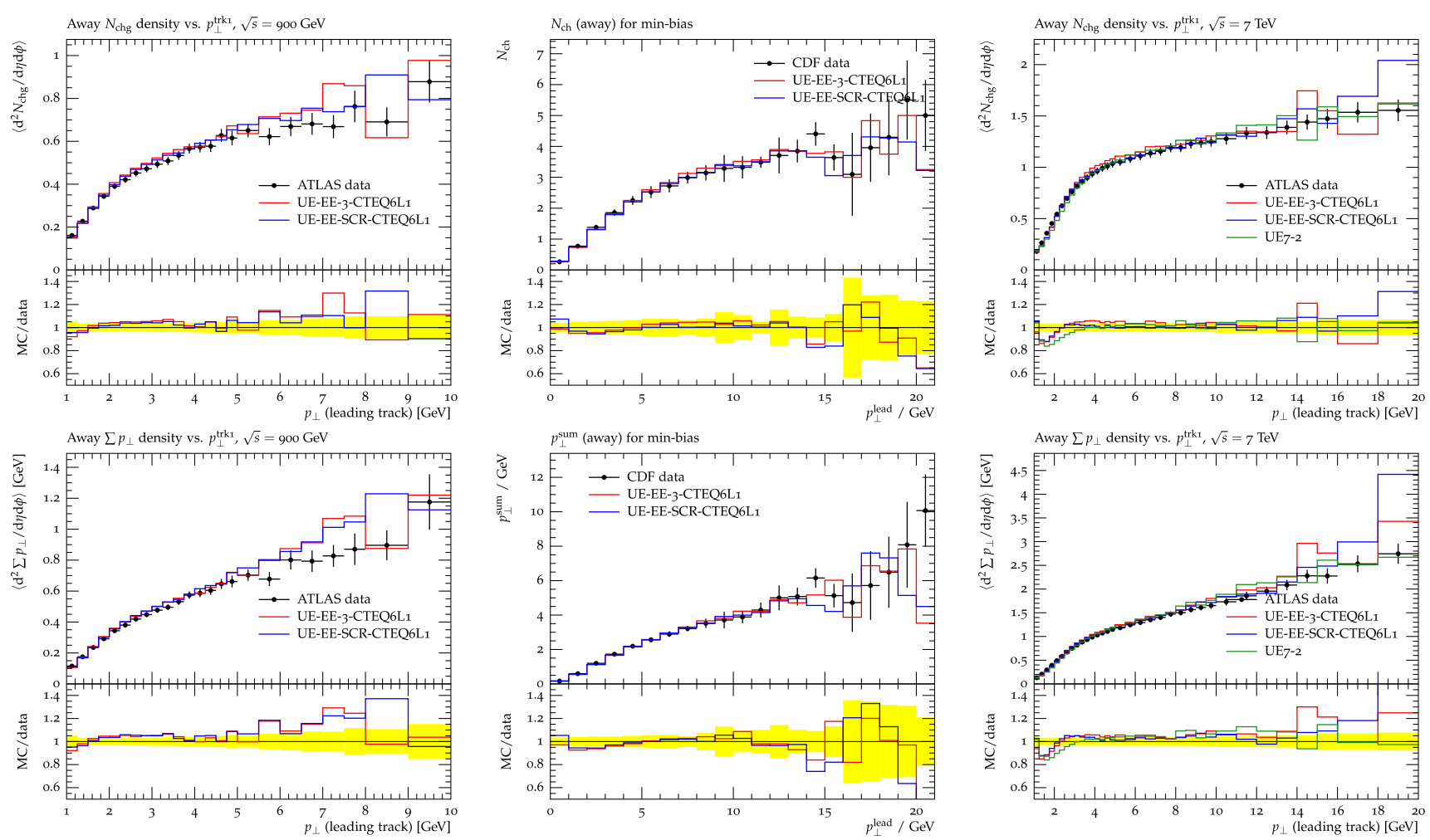

(a)

(b)

(c)

Fig. 14 Same as Fig. 13, but with the observables measured in the "away" region

Figure 18 shows the spread of the tuning results for each parameter against Professor's heuristic $\chi^{2}$. In the first row we present results for $900 \mathrm{GeV}$ and in the second row for $7 \mathrm{TeV}$. Each point is from a separate tune, made using various combinations of generator runs at different points in the parameter space. We see that the parameters are not well constrained and are sensitive to the input Monte Carlo (MC) runs. This is due to what we have already seen during the tuning of the MPI model without CR [23, 24, 60] to Tevatron data, namely the strong and constant correlation between $p_{\perp}^{\min }$ and $\mu^{2}$. This correlation reflects the fact that a smaller hadron radius always balances against a larger $p_{\perp}$ cutoff, as far as the underlying-event activity is concerned. With one of these two parameters fixed, the remaining parameters are much less sensitive to the input MC runs.

The most important information we can see on these figures is that the experimental data for the two different c.m. energies (900 GeV and $7 \mathrm{TeV}$ ) cannot be described by the same set of model parameters. More precisely, the experimental data prefers different $p_{\perp}^{\text {min }}$ values for different hadronic centre-of-mass energies, while the rest of the parameters may perhaps remain independent of the energy. This observation led us to the creation of energyextrapolated UE tunes, named UE-EE-3, in which all parameters are fixed except for $p_{\perp}^{\mathrm{min}}$, which varies with energy. We summarize the tune values for $p_{\perp}^{\min }$ at different energies in
Table 1. The other model parameters, which do not depend on the c.m. energy, are given in Table 2.

Since by construction the MPI model depends on the PDF set, we created two separate energy-extrapolated tunes for the CTEQ6L1 and MRST LO** PDFs. In general, both tunes yield similar and satisfactory descriptions of experimental data. ${ }^{2}$ As an example see Fig. 16, in which we compare the UE-EE-3 and UE-EE-3-CTEQ6L1 tunes to ATLAS UE observables, measured in all three regions (toward, transverse and away).

We repeated this procedure also for the SCR model. However, since in this case the tuning procedure was more complicated, as explained below, we concentrated on one PDF set only, namely CTEQ6L1. The first obvious complication was the larger number of parameters to tune. The second complication was associated with the fact that one of the tuning parameters, $N_{\text {steps }}$, is an integer number. The current version of Professor, however, does not provide such an option, instead it treats all parameters as real numbers. Therefore, we decided to carry out fifty separate tunes for different fixed values of $N_{\text {steps }}$, starting from 1 to 50 . The last problem that we encountered, which is probably associated

\footnotetext{
${ }^{2}$ The only difference is that the CTEQ6L1 gives more flexibility in the choice of the model parameters.
} 

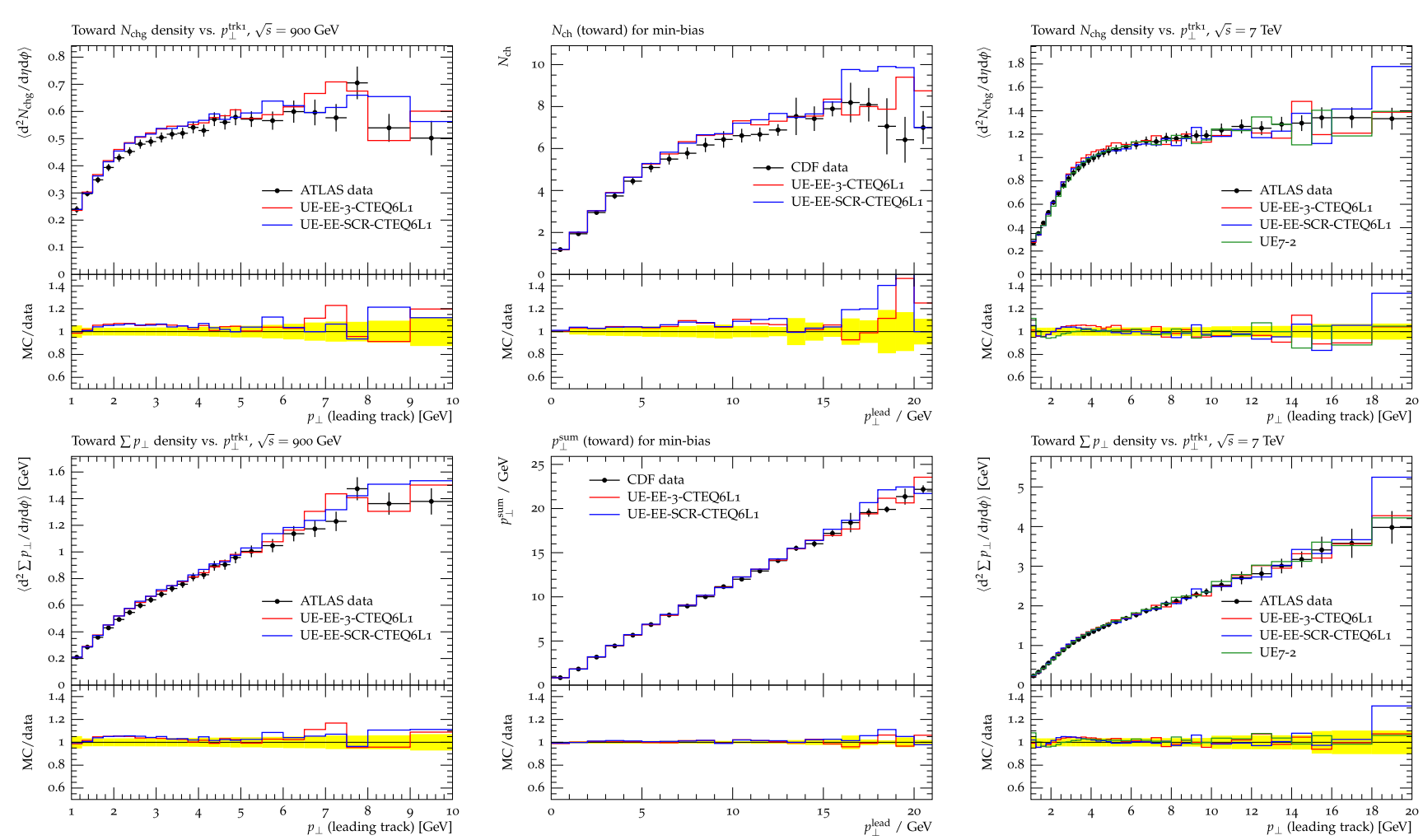

(a)

(b)

(c)

Fig. 15 Same as Fig. 13, but with the observables measured in the "toward" region

with the two previously mentioned problems, was that for some parameter values the predictions from Professor were significantly different from the results we received directly from HERWIG++ runs. Initially, we increased the order of the interpolating polynomials from second to fourth, which should improve Professor's predictions, but this did not improve the situation. Therefore, we first identified regions of the parameter space where this problem appeared most frequently and then excluded these from the tuning procedure. As a result, we obtained an energy-extrapolated underlyingevent tune for the SCR model, which we call UE-EE-SCRCTEQ6L1.

In Figs. 13, 14 and 15 we show a comparison of the PCR and SCR energy-extrapolated (CTEQ6L1) tunes and the UE7-2 tune against $\left\langle\mathrm{d}^{2} N_{\mathrm{ch}} / \mathrm{d} \eta \mathrm{d} \phi\right\rangle$ and $\left\langle\mathrm{d}^{2} \sum p_{t} / \mathrm{d} \eta \mathrm{d} \phi\right\rangle$ as a function of $p_{\perp}^{\text {lead }}$ for $p_{\perp}>500 \mathrm{MeV}$ in all three regions (toward, transverse and away) and at three different collider energies. We can see that the quality of the data description is high and at the same level for all tunes. Nevertheless, we favour the SCR model as here we have a clearer physics picture and a more flexible model.

In the last step, we parametrized the $p_{\perp}^{\min }$ dependence. In a first attempt we have chosen a logarithmic function to extrapolate $p_{\perp}^{\mathrm{min}}$ to energies different from the tune energies. Therefore we fitted a function of the form $p_{\perp}^{\min }(s)=$ $A \log (\sqrt{s} / B)$, where $A$ and $B$ are free fit parameters, to the three $p_{\perp}^{\text {min }}$ values obtained in the UE-EE-3 tune. The fit is shown in Fig. 19. Based on this, we provide UE tunes for c.m. energies the LHC was or will be operating at. Since the logarithmic form is not very stable for lower energies, we have replaced this ansatz with a power law, see also e.g. [61],

$p_{\perp}^{\min }(s)=p_{\perp, 0}^{\min }\left(\frac{\sqrt{s}}{E_{0}}\right)^{b}$.

This is the default parametrization of the energy dependence from HERWIG++ release 2.6 [62]. The default value of $E_{0}$ is $7 \mathrm{TeV}$. For the collider energies at consideration in our tunes there are no significant differences in all observables due to this change. The values for $b$ and $p_{\perp, 0}^{\min }$, which we find by fitting Eq. (7) to the $p_{\perp}^{\text {min }}$ values from Table 1, are summarized in the last two rows of Table 2.

For the preparation of the energy-extrapolated tunes we did not use any MB observables. Nevertheless, we show a comparison of the UE-EE-3-CTEQ6L1 and UE-EE-SCRCTEQ6L1 tunes to the diffraction-reduced ATLAS MB data at $7 \mathrm{TeV}$ (with $N_{\mathrm{ch}} \geq 6$ ) in Fig. 20. We see that the data is described slightly better by the SCR than by the PCR tune. Moreover, although these data sets were not taken into account in both tunes, the results are close to the experimental data. 

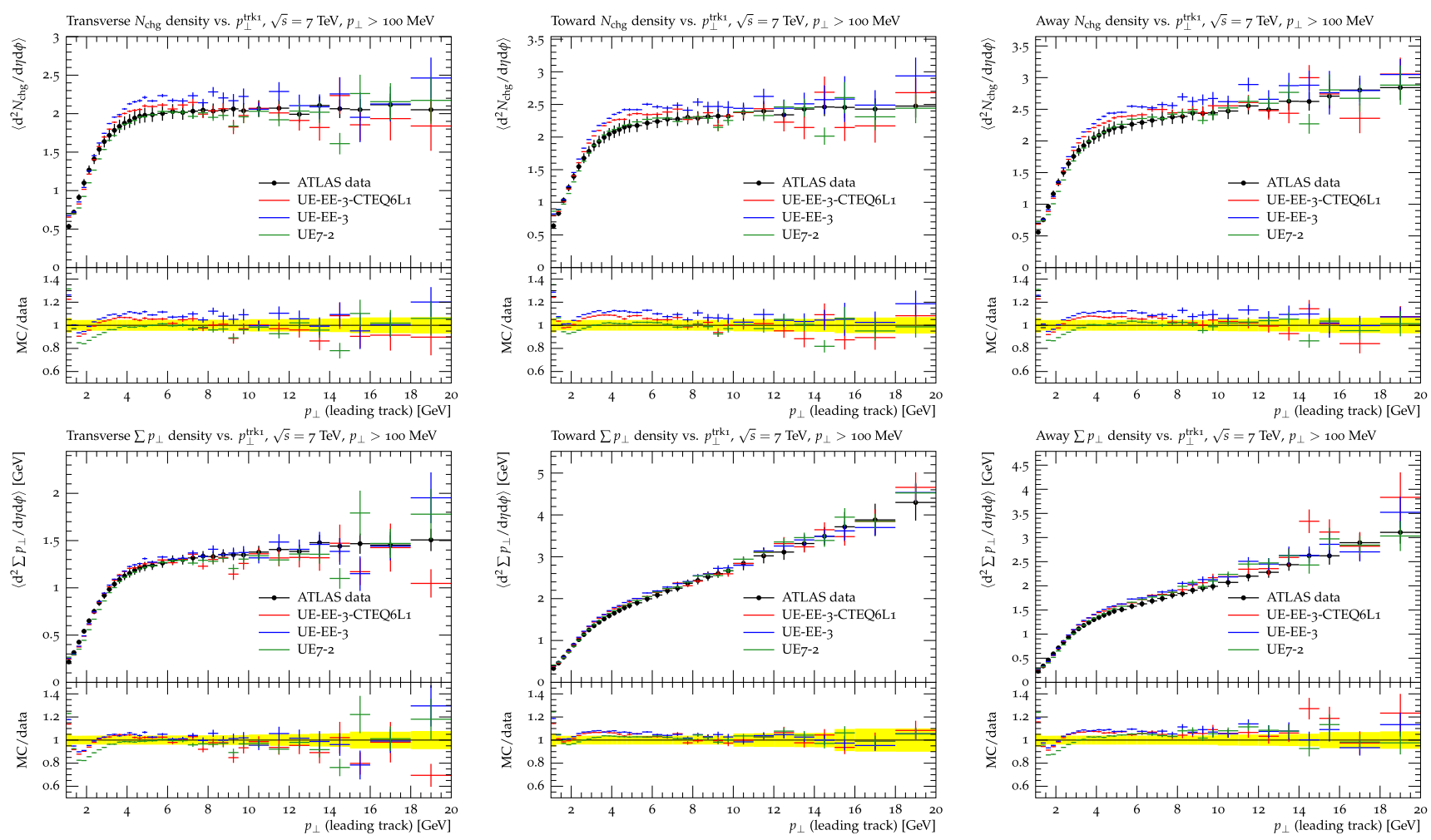

(a)

(b)

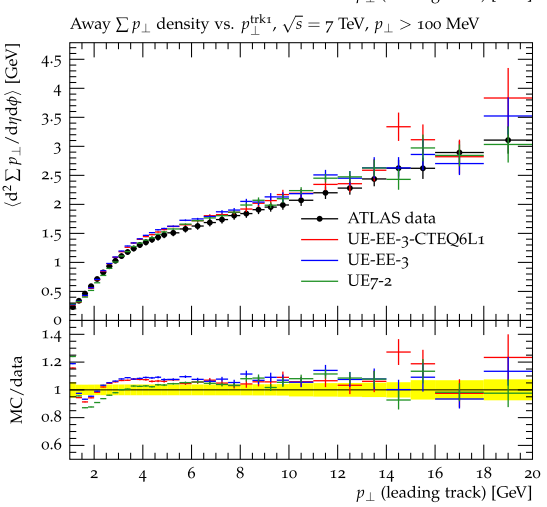

(c)

Fig. 16 ATLAS UE data at $7 \mathrm{TeV}$ for the lower $p_{\perp}$ cut $\left(p_{\perp}>\right.$ $100 \mathrm{MeV}$ ) for the transverse (1st column), towards ( 2 nd column) and away ( $3 r$ column) areas, showing the multiplicity density and $\sum p_{\perp}$

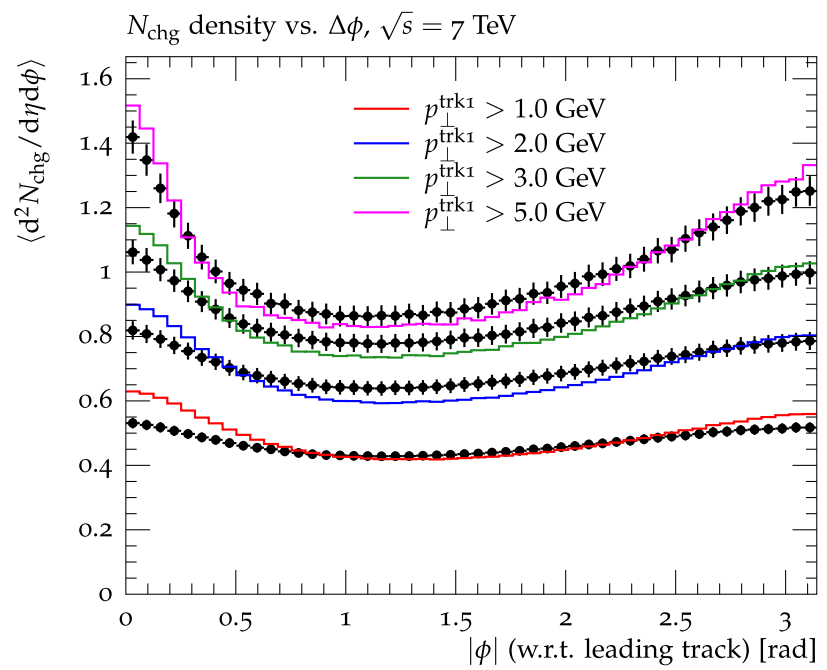

Fig. 17 Azimuthal distribution of the charged particle multiplicity (left panel) and $\sum p_{\perp}$ densities (right panel), with respect to the direction of the leading charged particle (at $\phi=0$ ), for $|\eta|<2.5$. The

In the future, we plan to study the energy scaling of the model parameters using diffraction-reduced minimum-bias data, and then, in more detail, the possibility of achieving a common description of the UE and MB data, cf. [63]. As of the charged particles as a function of $p_{\perp}^{\text {lead }}$. The data is compared to the UE7-2, UE-EE-3 and UE-EE-3-CTEQ6L 1 tunes

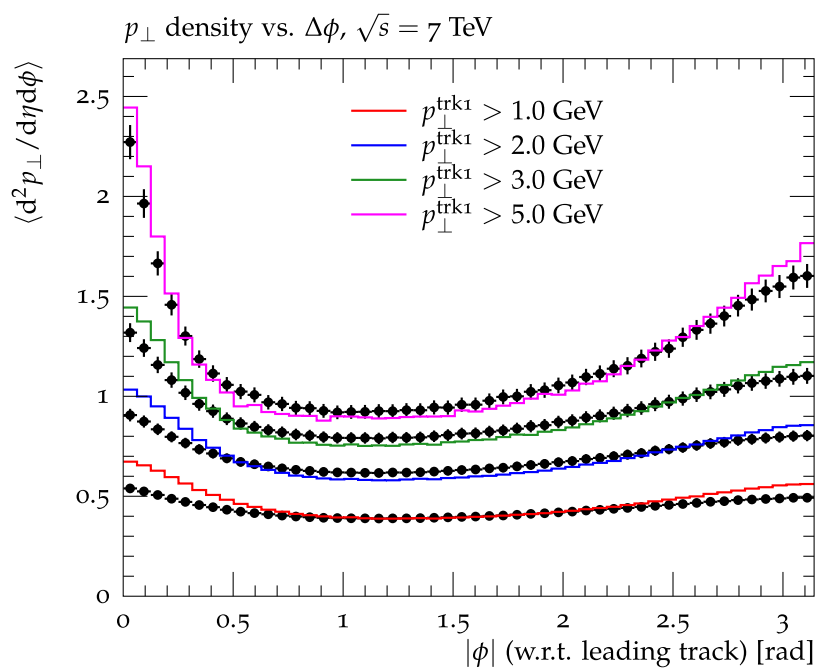

densities are shown for $p_{\perp}^{\text {lead }}>1 \mathrm{GeV}, p_{\perp}^{\text {lead }}>2 \mathrm{GeV}, p_{\perp}^{\text {lead }}>3 \mathrm{GeV}$ and $p_{\perp}^{\text {lead }}>5 \mathrm{GeV}$. The data is compared to the UE7-2 tune

can be seen in Fig. 21, the UE tunes fail to reproduce the ATLAS MB data at $7 \mathrm{TeV}$ with a less tight cut on the number of charged particles, $N_{\mathrm{ch}} \geq 2$, and where all charged particles with $p_{\perp}>100 \mathrm{MeV}$ are taken into account. This is not 

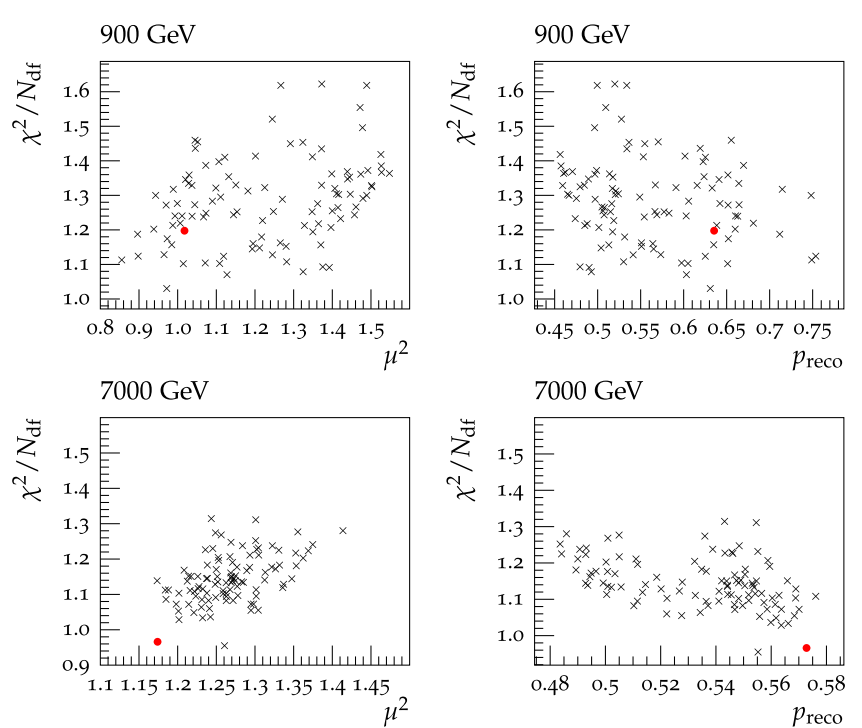

Fig. 18 The spread of UE-EE-3-CTEQ6L1 tuning results for the parameters $\mu^{2}, p_{\text {reco }}, p_{\text {disrupt }}$ and $p_{\perp}^{\min }$, using cubic generator response parametrizations with all generator runs (circles) and with subsets of

Table 1 Tune values for $p_{\perp}^{\min }$. All other model parameters, which do not depend on the c.m. energy, are summarized in Table 2

\begin{tabular}{llll}
\hline$p_{\perp}^{\min }[\mathrm{GeV}]$ & & & \\
\hline$\sqrt{s}[\mathrm{GeV}]$ & 900 & 1800 & 7000 \\
\hline UE-EE-3 & 1.55 & 2.26 & 2.75 \\
UE-EE-3-CTEQ6L1 & 1.86 & 2.55 & 3.06 \\
UE-EE-SCR-CTEQ6L1 & 1.58 & 2.14 & 2.60 \\
\hline
\end{tabular}

Table 2 Parameters of the energy-extrapolating underlying-event tunes. The last two parameters describe the running of $p_{\perp}^{\mathrm{min}}$ according to Eq. (7)

\begin{tabular}{llll}
\hline & UE-EE-3 & UE-EE-3-CTEQ6L1 & UE-EE-SCR-CTEQ6L1 \\
\hline$\mu^{2}\left[\mathrm{GeV}^{2}\right]$ & 1.11 & 1.35 & 1.5 \\
$p_{\text {disrupt }}$ & 0.80 & 0.75 & 0.8 \\
$p_{\text {reco }}$ & 0.54 & 0.61 & - \\
$c$ & - & - & 0.01 \\
$f$ & - & - & 0.21 \\
$N_{\text {steps }}$ & - & - & 10 \\
$\alpha$ & - & - & 0.66 \\
$p_{\perp, 0}^{\min }[\mathrm{GeV}]$ & 3.11 & 2.81 & 2.64 \\
$b$ & 0.21 & 0.24 & 0.21 \\
\hline
\end{tabular}

surprising, however, since HERWIG lacks a model for soft diffractive physics so far. That explains the poor description of both the charged multiplicity and the average transverse momentum in the low-multiplicity bins. On the other hand, the unsatisfactory description of the shown observables in
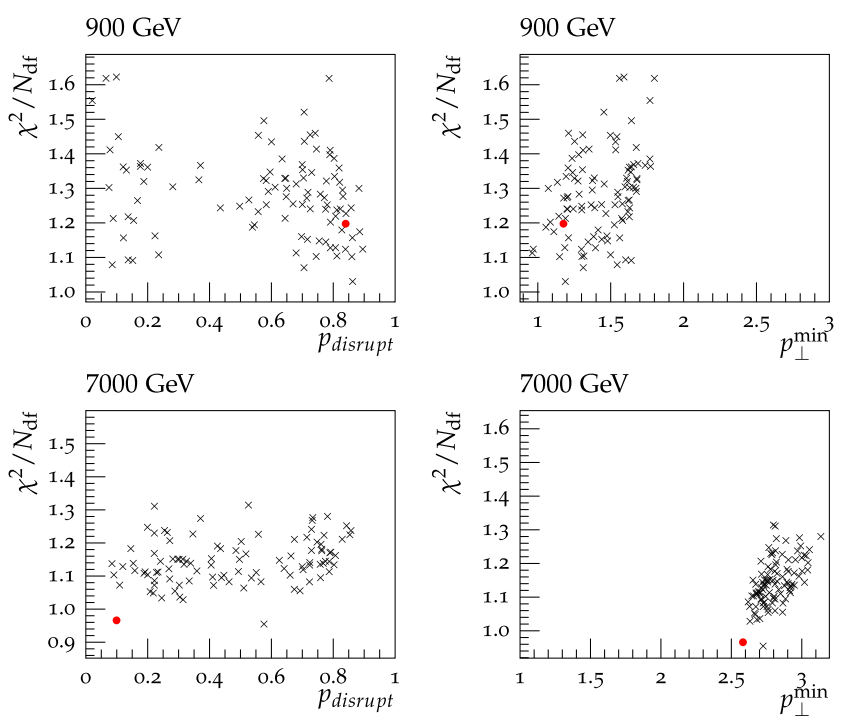

generator runs (crosses). The first row shows results for tunes to data at $900 \mathrm{GeV}$ and the second at $7 \mathrm{TeV}$

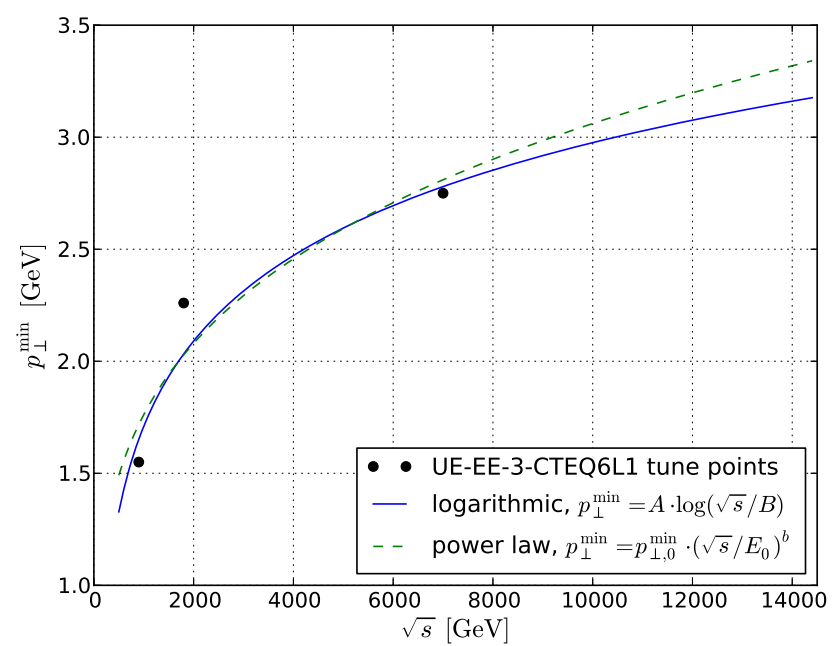

Fig. 19 Energy extrapolation of $p_{\perp}^{\min }$ in the UE-EE-3-CTEQ6L1 tune

the high multiplicity tail may indicate missing physics in the model. It might, however, as well be resolved by a dedicated MB tune. Both possibilities are left for future work. In particular, we point out the lack of an explicit model for diffractive events. A more complete description of the MB data should also include a modelling of these.

\section{Conclusions}

We have introduced two different models for non-perturbative colour reconnections in HERWIG. The models are of slightly different computational complexity but give very similar results. The tuning results have shown that the SCR 

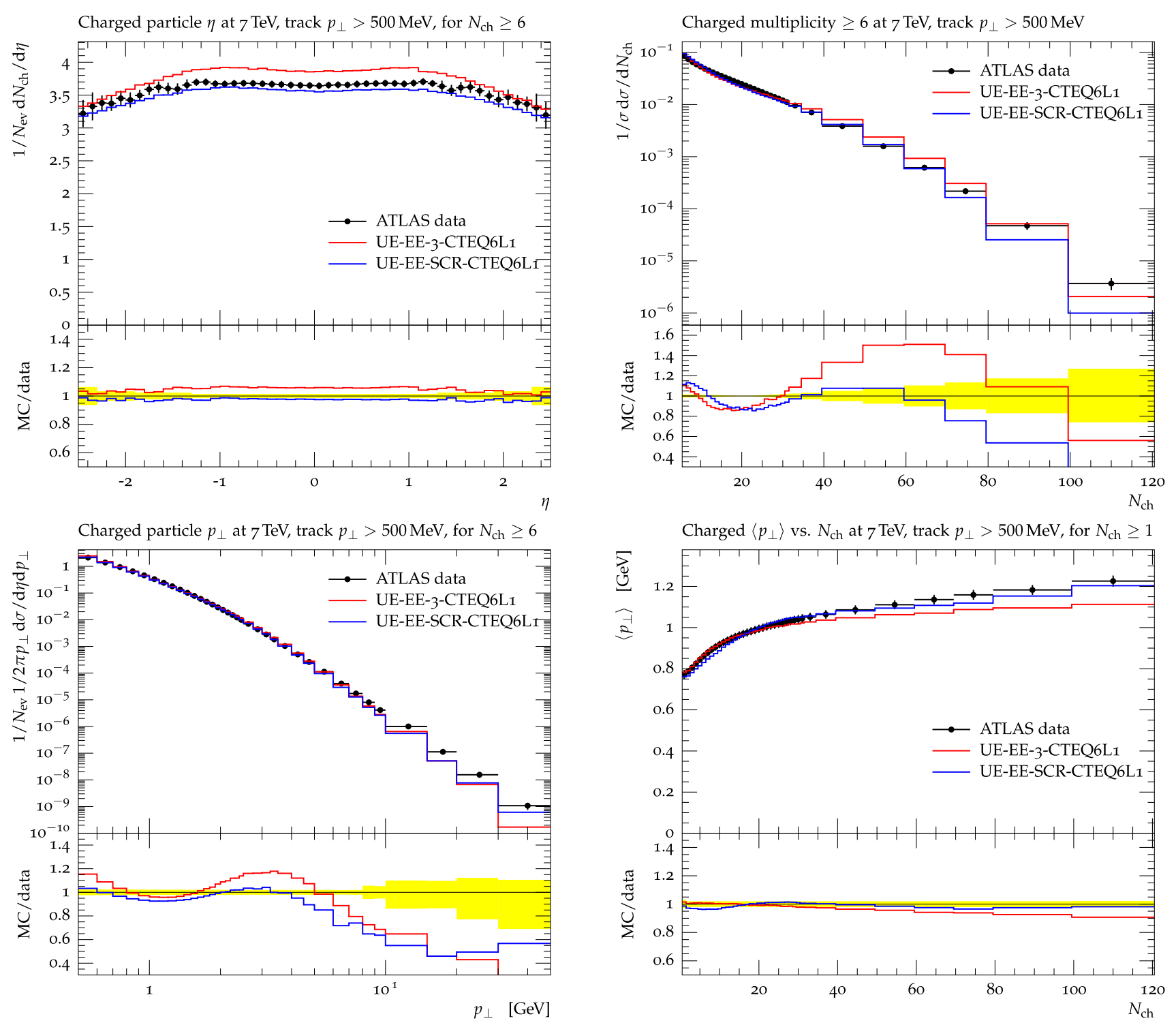

Fig. 20 Comparison of the UE-EE-3-CTEQ6L1 and UE-EE-SCR-CTEQ6L1 tunes to ATLAS minimum-bias distributions at $\sqrt{s}=7$ TeV, with $N_{\text {ch }} \geq 6, p_{\perp}>500 \mathrm{MeV}$ and $|\eta|<2.5$

is preferred to have parameters that force a quick 'cooling' of the system and therefore results in a very similar model evolution as in the simpler PCR model. We therefore consider the PCR as a special case of the SCR model for quick cooling and keep the SCR as the more flexible model for future versions of HERWIG++. As a consequence, we understand that the data demands a final state that does not obey a perfectly minimized colour length. We interpret this as a model limitation. At some point the picture of colour lines breaks down. Colour lines themselves are only a valid prescription up to leading order in the $N_{C} \rightarrow \infty$ limit. Furthermore, the mechanism addresses the non-perturbative regime where the picture of the colour triplet charges themselves is already a model by itself and possibly completely washed out.
We have studied the mechanism of colour reconnection in detail and found that in fact the non-perturbative parts of the simulation demand the colour reconnection mechanism in order to repair the lack of information on the colour flow. The intuitive picture we have based our model on could be verified. The idea of colour preconfinement is meaningful in the context of the hadronization model and has to be rectified when a model of multiple partonic interactions is applied without further information on the colour structure in between the multiple scatters.

Furthermore, we have shown that by tuning the MPI model with CR we can obtain a proper description of nondiffractive MB ATLAS observables. We present the energyextrapolated tune UE-EE-3, which is an important step to- 

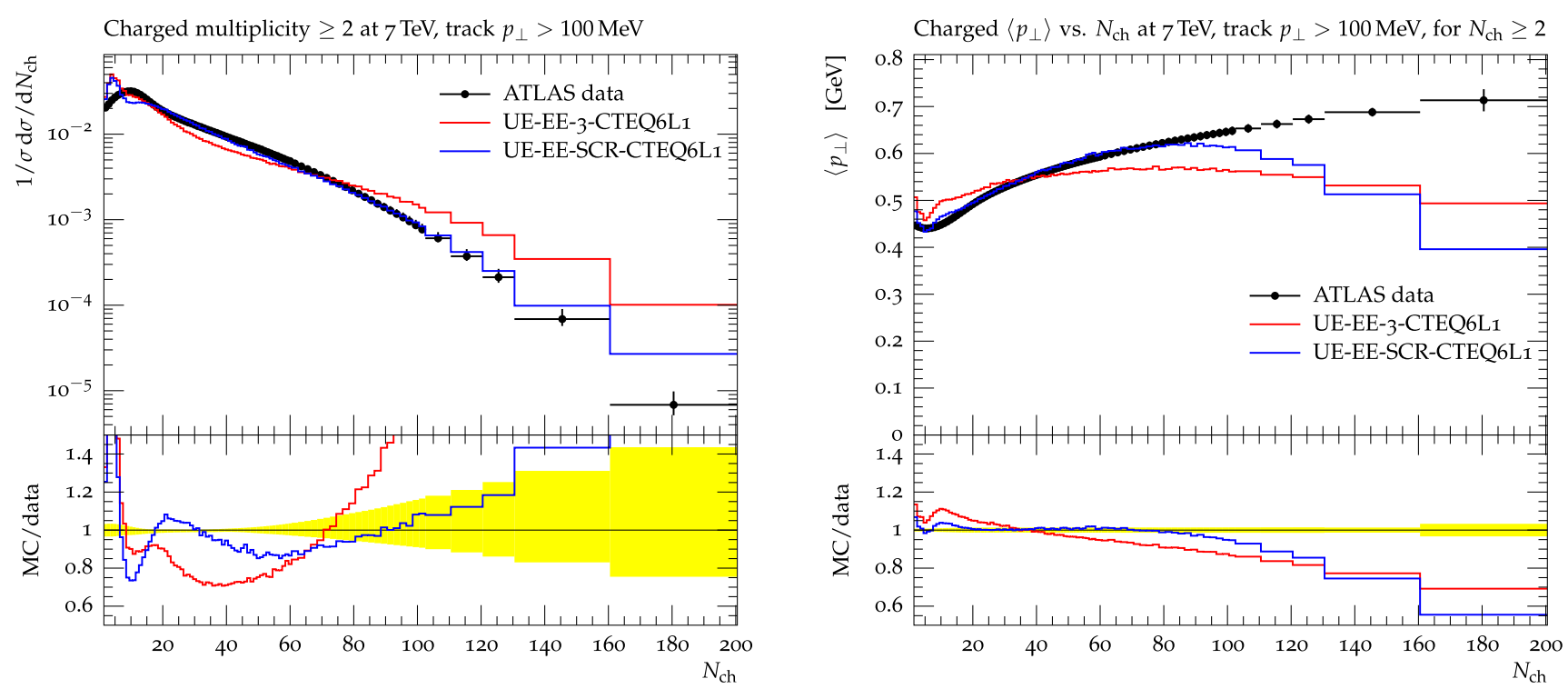

Fig. 21 Comparison of underlying-event tunes to presumably diffraction-enhanced MB observables, measured by ATLAS at $\sqrt{s}=7$ TeV, with $N_{\mathrm{ch}} \geq 2, p_{\perp}>100 \mathrm{MeV}$ and $|\eta|<2.5$

wards the understanding of the energy dependence of the model. Finally, we have unified the different tunes of the MPI model in HERWIG++ into a simple parametrization of the $p_{\perp}^{\min }$ dependence in a way that allows us to describe data at different energies with only one set of parameters. News concerning HERWIG tunes are available on the tune wiki page [50].

Acknowledgements We are grateful to the other members of the HERWIG collaboration for critical discussions and support. We acknowledge financial support from the Helmholtz Alliance "Physics at the Terascale". This work was funded in part (AS) by the LancasterManchester-Sheffield Consortium for Fundamental Physics under STFC grant ST/J000418/1.

Open Access This article is distributed under the terms of the Creative Commons Attribution License which permits any use, distribution, and reproduction in any medium, provided the original author(s) and the source are credited.

\section{References}

1. A.A. Affolder et al. (CDF Collaboration), Phys. Rev. D 65, 092002 (2002)

2. T. Aaltonen et al. (CDF Collaboration), Phys. Rev. D 82, 034001 (2010)

3. G. Aad et al. (ATLAS Collaboration), Phys. Lett. B 688, 21 (2010)

4. G. Aad et al. (ATLAS Collaboration), Phys. Rev. D 83, 112001 (2011)

5. G. Aad et al. (ATLAS Collaboration), New J. Phys. 13, 053033 (2011)

6. G. Aad et al. (ATLAS Collaboration), Eur. Phys. J. C 71, 1636 (2011)

7. V. Khachatryan et al. (CMS Collaboration), J. High Energy Phys. 1002, 041 (2010)
8. V. Khachatryan et al. (CMS Collaboration), Phys. Rev. Lett. 105, 022002 (2010)

9. V. Khachatryan et al. (CMS Collaboration), Phys. Lett. B 699, 48 (2011)

10. S. Chatrchyan et al. (CMS Collaboration), J. High Energy Phys. 1109, 109 (2011)

11. K. Aamodt et al. (ALICE Collaboration), Eur. Phys. J. C 68, 89 (2010)

12. K. Aamodt et al. (ALICE Collaboration), Eur. Phys. J. C 68, 345 (2010)

13. K. Aamodt et al. (ALICE Collaboration), Phys. Lett. B 693, 53 (2010)

14. M. Bähr et al., Eur. Phys. J. C 58, 639 (2008)

15. T. Sjöstrand, S. Mrenna, P. Skands, J. High Energy Phys. 05, 026 (2006)

16. T. Sjöstrand, S. Mrenna, P. Skands, Comput. Phys. Commun. 178, $852(2008)$

17. T. Gleisberg et al., J. High Energy Phys. 02, 007 (2009)

18. T. Sjöstrand, M. van Zijl, Phys. Rev. D 36, 2019 (1987)

19. J.M. Butterworth, J.R. Forshaw, M.H. Seymour, Z. Phys. C 72, 637 (1996)

20. V. Khoze, F. Krauss, A. Martin, M. Ryskin, K. Zapp, Eur. Phys. J. C 69, 85 (2010)

21. T. Sjöstrand, P.Z. Skands, J. High Energy Phys. 03, 053 (2004)

22. T. Sjöstrand, P.Z. Skands, Eur. Phys. J. C 39, 129 (2005)

23. M. Bähr, S. Gieseke, M.H. Seymour, J. High Energy Phys. 07, 076 (2008)

24. M. Bähr, J.M. Butterworth, S. Gieseke, M.H. Seymour, 0905.4671 (2009)

25. I. Borozan, M.H. Seymour, J. High Energy Phys. 09, 015 (2002)

26. S. Gieseke, M.H. Seymour, A. Siódmok, J. High Energy Phys. 06, 001 (2008)

27. J. Pumplin et al., J. High Energy Phys. 07, 012 (2002)

28. A. Sherstnev, R.S. Thorne, Eur. Phys. J. C 55, 553 (2008)

29. S. Gieseke, S. Plätzer, C. Röhr, A. Siódmok, DESY-PROC-201001 (2010). Available from http://plhc2010.desy.de/proceedings

30. S. Gieseke, C. Röhr, A. Siódmok, 1110.2675 (2011)

31. P. Bartalini et al., 1111.0469 (2011)

32. D. Amati, G. Veneziano, Phys. Lett. B 83, 87 (1979)

33. M. Sandhoff, P.Z. Skands, hep-ph/0604120 (2005) 
34. P.Z. Skands, D. Wicke, Eur. Phys. J. C 52, 133 (2007)

35. D. Wicke, P.Z. Skands, Nuovo Cimento B 123, S1 (2008)

36. B.R. Webber, Nucl. Phys. B 238, 492 (1984)

37. G. 't Hooft, Nucl. Phys. B 72, 461 (1974)

38. S. Gieseke, A. Ribon, M.H. Seymour, P. Stephens, B. Webber, J. High Energy Phys. 02, 005 (2004)

39. S. Gieseke et al., 1102.1672 (2011)

40. S. Kirkpatrick, C.D. Gelatt, M.P. Vecchi, Science 220, 671 (1983)

41. G. Abbiendi et al. (OPAL Collaboration), Eur. Phys. J. C 40, 287 (2005)

42. G. Abbiendi et al. (OPAL Collaboration), Eur. Phys. J. C 20, 601 (2001)

43. K. Ackerstaff et al. (OPAL Collaboration), Eur. Phys. J. C 7, 369 (1999)

44. P. Pfeifenschneider et al. (JADE Collaboration), Eur. Phys. J. C 17, 19 (2000)

45. P. Abreu et al. (DELPHI Collaboration), Z. Phys. C 67, 543 (1995)

46. P. Abreu et al. (DELPHI Collaboration), Z. Phys. C 73, 11 (1996)

47. R. Barate et al. (ALEPH Collaboration), Phys. Rep. 294, 1 (1998)

48. D. Decamp et al. (ALEPH Collaboration), Phys. Lett. B 273, 181 (1991)

49. A. Heister et al. (ALEPH Collaboration), Eur. Phys. J. C 35, 457 (2004)

50. Herwig++ Collaboration, http://herwig.hepforge.org/, http:// herwig.hepforge.org/trac/wiki/MB_UE_tunes
51. P. Skands, A. Karneyeu, D. Konstantinov, M. Mangano, L. Mijovic, W. Pokorski, S. Prestel, A. Pytel, http://mcplots.cern.ch/

52. J. Abdallah et al. (DELPHI Collaboration), Eur. Phys. J. C 51, 249 (2007)

53. P. Achard et al. (L3 Collaboration), Phys. Lett. B 561, 202 (2003)

54. T. Ziegler (ALEPH Collaboration), ALEPH-2001-047

55. G. Abbiendi et al. (OPAL Collaboration), Eur. Phys. J. C 45, 291 (2006)

56. D. Duchesneau, New method based on energy and particle flow in $e^{+} e^{-} \rightarrow W^{+} W^{-} \rightarrow$ hadron events for color reconnection studies. LAPP-EXP-2000-02 (2000)

57. D. Acosta et al. (CDF Collaboration), Phys. Rev. D 70, 072002 (2004)

58. A. Buckley et al., 1003.0694 (2010)

59. A. Buckley, H. Hoeth, H. Lacker, H. Schulz, J.E. von Seggern, Eur. Phys. J. C 65, 331 (2010)

60. M. Bähr, J.M. Butterworth, M.H. Seymour, J. High Energy Phys. 01, 065 (2009)

61. M. Ryskin, A. Martin, V. Khoze, Eur. Phys. J. C 71, 1617 (2011)

62. K. Arnold et al., 1205.4902 (2012)

63. H. Schulz, P. Skands, Eur. Phys. J. C 71, 1644 (2011) 